
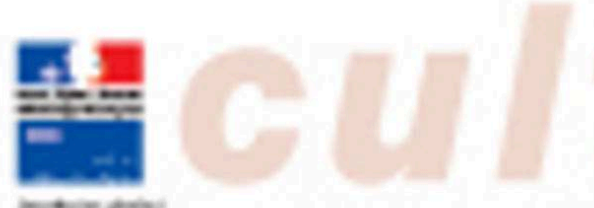

$2+\sin 20$

inisis

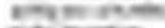

$+2+\infty$

simen

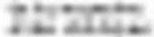

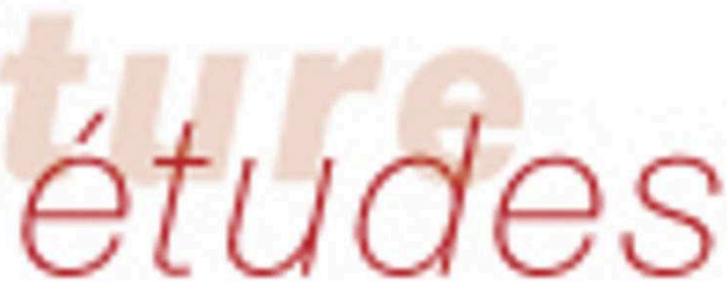

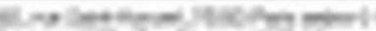

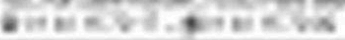

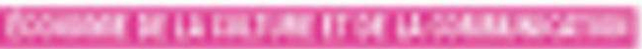

\title{
Territoires et resseurees des compagnies en France
}

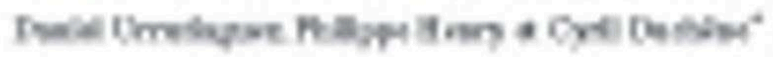

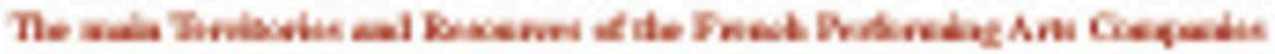

\section{Masi prove}

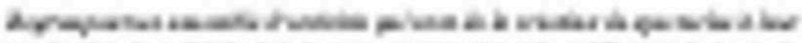

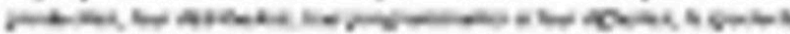

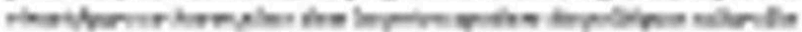

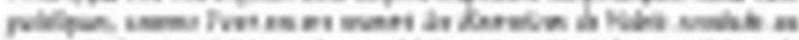

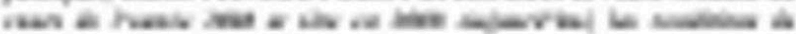

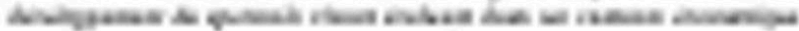

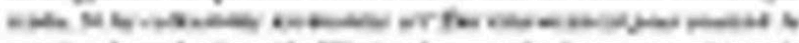

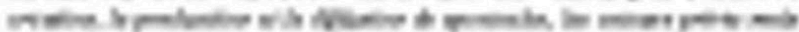

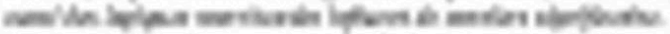

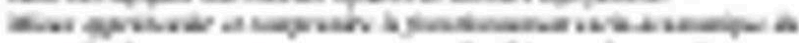

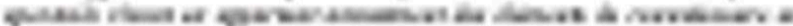

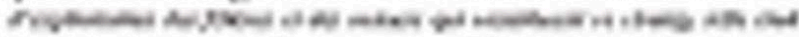

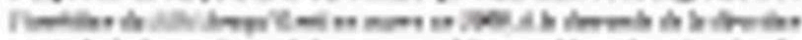

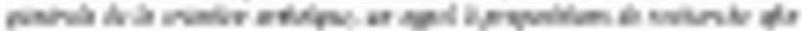

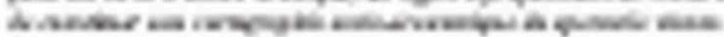

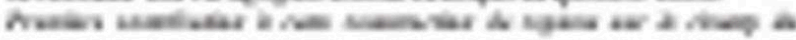

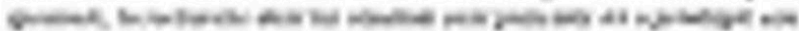

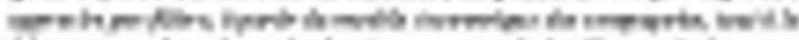

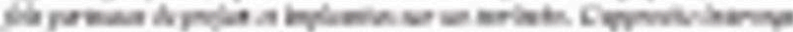

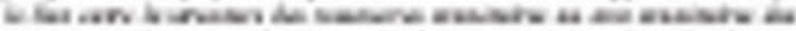

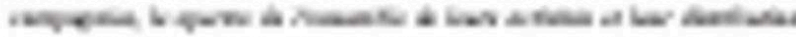

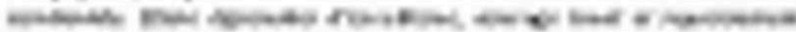

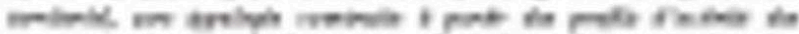

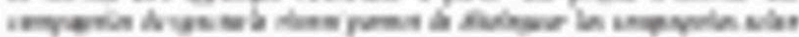

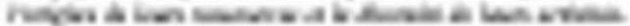

Novjebstross ous

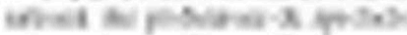

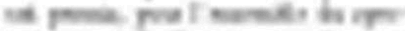

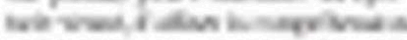

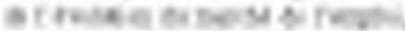

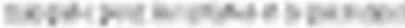

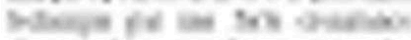

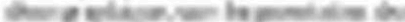

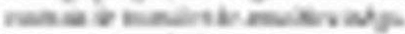

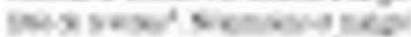

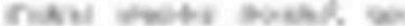

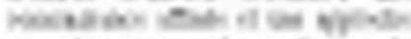

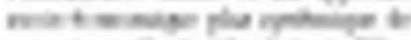

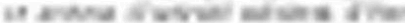

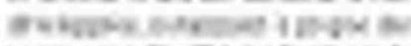

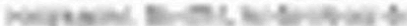

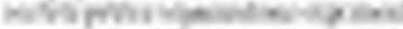

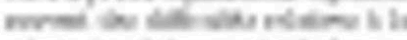

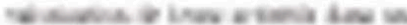

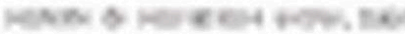

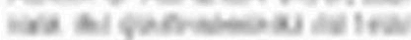

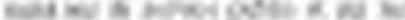

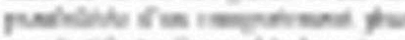

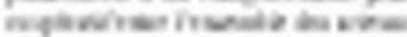

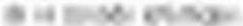

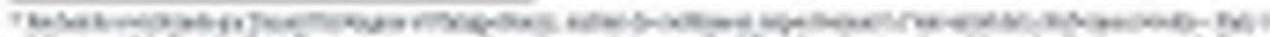

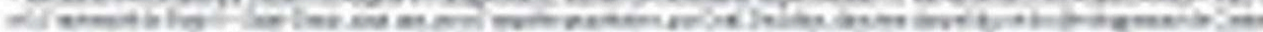

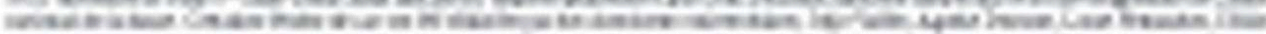

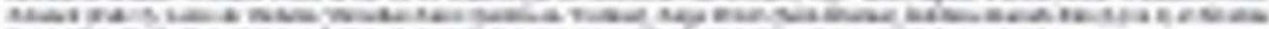

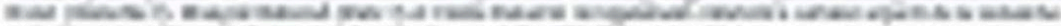

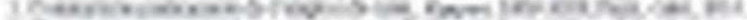

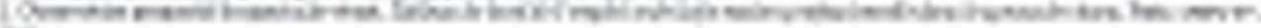
30
} 


\section{Territoires et ressources des compagnies en France}

The main Territories and Resources of the French Performing Arts Companies

Daniel Urrutiaguer, Philippe Henry et Cyril Duchêne

Éditeur : Département des études, de la prospective et des statistiques

Lieu d'édition : Paris

Année d'édition : 2012

Date de mise en ligne : 8 juillet 2015

Collection : Culture études

ISBN électronique : 9782111399068

\section{Sbooks}

http://books.openedition.org

Édition imprimée

Date de publication : 1 mars 2012

Nombre de pages : 16

Référence électronique

URRUTIAGUER, Daniel; HENRY, Philippe ; et DUCHÊNE, Cyril. Territoires et ressources des compagnies en France. Nouvelle édition [en ligne]. Paris : Département des études, de la prospective et des statistiques, 2012 (généré le 25 avril 2021). Disponible sur Internet : <http://books.openedition.org/ deps/151>. ISBN : 9782111399068 

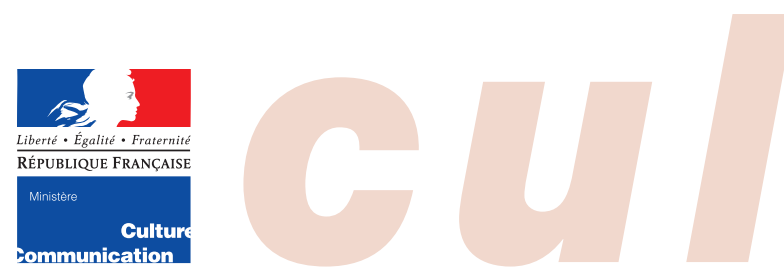

Secrétariat général

Service de la

coordination des

politiques culturelles

et de l'innovation

Département

des études,

de la prospective

et des statistiques

\title{
Territoires et ressources des compagnies en France
}

\author{
Daniel Urrutiaguer, Philippe Henry et Cyril Duchêne*
}

\author{
The main Territories and Resources of the French Performing Arts Companies
}

\begin{abstract}
Avant-propos
Regroupant un ensemble d'activités qui vont de la création de spectacles à leur production, leur distribution, leur programmation et leur diffusion, le spectacle vivant figure en bonne place dans les préoccupations des politiques culturelles publiques, comme l'ont encore montré les Entretiens de Valois conduits au cours de l'année 2008 et clos en 2009. Aujourd'hui, les conditions de développement du spectacle vivant évoluent dans un contexte économique tendu. Si les collectivités territoriales et l'État interviennent pour soutenir la création, la production et la diffusion de spectacles, les acteurs privés mais aussi des logiques marchandes influent de manière significative.

Mieux appréhender et comprendre le fonctionnement socio-économique du spectacle vivant en apportant notamment des éléments de connaissance et d'explicitation des filières et des acteurs qui constituent ce champ, telle était l'ambition du DEPS lorsqu'il mit en ouvre en 2009, à la demande de la direction générale de la création artistique, un appel à propositions de recherche afin de constituer une cartographie socio-économique du spectacle vivant.

Première contribution à cette construction de repères sur le champ du spectacle, la recherche dont les résultats sont présentés ici a privilégié une approche par filière, à partir du modèle économique des compagnies, tout à la fois porteuses de projets et implantées sur un territoire. L'approche interroge le lien entre la structure des ressources monétaires ou non monétaires des compagnies, le spectre de l'ensemble de leurs activités et leur distribution territoriale. Entre injonction d'excellence, ancrage local et rayonnement territorial, une typologie construite à partir des profils d'activité des compagnies de spectacle vivant permet de distinguer les compagnies selon l'origine de leurs ressources et la diversité de leurs activités.
\end{abstract}

Guillaume BoudY
Depuis 2003, les travaux du Conseil national des professions du spectacle ont permis, pour l'ensemble du spectacle vivant, d'affiner la compréhension de l'évolution du marché de l'emploi, marquée pour les artistes et le personnel technique par une forte croissance démographique, une fragmentation des contrats de travail et de sensibles inégalités de revenus ${ }^{1}$. Néanmoins et malgré d'autres avancées récentes ${ }^{2}$, une connaissance affinée et une approche socio-économique plus systémique de ce secteur d'activité méritent d'être développées, notamment à propos des compagnies. En effet, les directions de ces très petites organisations expriment souvent des difficultés relatives à la valorisation de leurs activités dans un contexte de concurrence accrue, mais aussi des questionnements sur leurs missions de service public et sur les possibilités d'un comportement plus coopératif entre l'ensemble des acteurs de ce monde artistique.

\footnotetext{
* Recherche coordonnée par Daniel Urrutiaguer et Philippe Henry, maîtres de conférences respectivement à l'université de la Sorbonne nouvelle - Paris 3 et à l'université de Paris 8 - Saint-Denis, ainsi que, pour l'enquête quantitative, par Cyril Duchêne, directeur des publics et du développement du Centre national de la danse. Certaines études de cas ont été réalisées par des chercheurs universitaires, Julie Valéro, Agathe Dumont, Laure Fernandez, Cécile Schenck (Paris 3), Laure de Verdalle (Versailles-Saint-Quentin-en-Yvelines), Serge Proust (Saint-Étienne), Bérénice Hamidi-Kim (Lyon 2) et Séverine Ruset (Grenoble 3). François Rémond (Paris 3) et Cécile Delassus ont également collaboré à certains aspects de la recherche.

1. Commission permanente de l'emploi du CNPS, Rapport 2008-2009, Paris, CNPS, 2010.

2. Observatoire prospectif du spectacle vivant, Tableau de bord de l'emploi et de la formation professionnelle dans le spectacle vivant, Paris, OMPQ-Sv, 2011.
} 
Explorer les liens entre la distribution territoriale des activités des compagnies théâtrales et chorégraphiques en France et la structure de leurs ressources, monétaires et non monétaires, se révèle une entrée pertinente pour préciser les défis économiques, politiques, artistiques et sociaux auxquels ces organisations sont exposées. La méthodologie s'appuie sur un échantillon de 51 études de cas qualitatives et le traitement quantitatif de 572 réponses à un questionnaire ${ }^{3}$.

Les références à la littérature disponible sur le champ étudié permettent d'articuler les résultats inédits à des confirmations de faits déjà connus.

\section{UN PAYSAGE PRÉCISÉ}

\section{Une typologie inédite des compagnies}

Au-delà de la singularité de toute démarche artistique, trois variables principales ont été dégagées et permettent de mieux comprendre les interactions entre les compagnies et leur environnement, dans le cadre d'une analyse de filière du spectacle vivant:

1) le centre de gravité territorial de la diffusion des spectacles des compagnies, et plus particulièrement l'importance relative de leur région d'appartenance ou d'implantation;

2) le niveau de reconnaissance institutionnelle des établissements d'accueil des activités, et plus particulièrement l'importance relative des établissements artistiques ou culturels labellisés par le ministère de la Culture et de la Communication au titre de la production ou de la diffusion artistiques (théâtres nationaux, centres dramatiques ou chorégraphiques nationaux, festivals internationaux ; scènes nationales, scènes conventionnées, festivals nationaux); la corrélation entre cette variable et la précédente reflète les liens entre la capacité d'excentration de la diffusion d'une compagnie hors de sa région d'appartenance ou d'implantation et son degré de programmation des lieux de spectacles labellisés ;

3) le niveau du budget annuel des compagnies.

Ces trois variables se sont révélées beaucoup plus discriminantes que la différenciation usuelle selon la discipline $^{4}$ ou encore selon le genre des directeurs(rices) artistiques. Quatre groupes principaux sont distinguables, selon l'importance respective de la région du siège social et des autres territoires, national ou international ${ }^{5}$, dans le temps consacré à la diffusion. L'approche qualitative, confirmée par l'échantillon quantitatif, a ainsi permis de différencier : - les compagnies dites « régionales », dont l'activité se réalise exclusivement ou presque sur leur territoire régional d'appartenance ( $90 \%$ ou plus des représentations de spec- tacles). L'activité se déroule également presque exclusivement dans des établissements ne disposant d'aucun label national du ministère de la Culture et de la Communication (centres artistiques et culturels municipaux, festivals régionaux ou off d'Avignon) et dans des établissements dont la vocation première n'est pas artistique (écoles, maisons des jeunes et de la culture ou maisons de quartier, bibliothèques, hôpitaux, prisons...);

- les compagnies dites « transrégionales », dont l'activité de diffusion de spectacles reste majoritairement située dans la région d'implantation ( $55 \%$ à $75 \%$ de la programmation), cette première caractéristique se complétant d'un taux de diffusion très important dans les établissements non labellisés par le ministère (de $70 \%$ à $80 \%$ des spectacles de la compagnie). Une part de plus en plus significative de leur activité dépend néanmoins des partenariats qu'elles parviennent à nouer hors de leur région d'origine ou d'appartenance actuelle, et de ceux qu'elles concrétisent avec les établissements à label national - d'abord de production - ( $7 \%$ à $28 \%$ des représentations) ;

- les compagnies dites «multirégionales », dont la part d'activité hors région d'implantation l'emporte (55\% à $80 \%$ des représentations de spectacles au-delà de la région d'appartenance), même si cette dernière demeure importante pour le projet artistique et culturel de ces compagnies. Ces compagnies déclarent toujours des représentations à l'étranger (de 1 à un peu moins de 10 représentations). Enfin, la part des représentations réalisées dans les établissements à label national est à peine plus importante que pour les compagnies transrégionales, les établissements non labellisés par le ministère continuant à être prédominants pour l'activité de ces compagnies ;

- les compagnies dites «excentrées », dont l'essentiel des activités se déroule hors de la région du siège social et administratif (au moins $70 \%$ des représentations de spectacles programmées hors de cette région); les établissements artistiques labellisés par le ministère (au titre de la production ou de la diffusion) sont les principaux partenaires de ces compagnies, sans pour autant être exclusifs ; les lieux non labellisés ou à vocation première autre qu'artistique apparaissent ainsi souvent de manière non négligeable (de $9 \%$ à $39 \%$ des représentations).

Sur le plan des disciplines artistiques, on note une surreprésentation du théâtre et du conte dans les profils « régionaux », alors que les arts du cirque, de la marionnette et de la rue sont plus présents dans les autres groupes.

Le nombre restreint de compagnies relevant du dernier groupe $(8 \%)$ doit être souligné, d'autant qu'il correspond à l'idéal encore très largement répandu d'une excellence artistique d'abord préoccupée de rayonnement national ou international, plus que d'attaches territoriales. Les trois autres groupes, où se conjuguent diversement mais constamment une visée de qualité artistique et une inscription ter-

\footnotetext{
3. Voir encadré méthodologique, p. 16.

4. Disciplines représentées dans les échantillons : théâtre, danse, cirque, arts de la rue, marionnettes, conte. Un nombre non négligeable de compagnies se présentent aussi comme pluridisciplinaires.

5. Les résultats quantitatifs s'appuient sur les 503 troupes ayant classé la diffusion de spectacle dans leurs quatre activités principales. L'information fournie par les 69 autres compagnies qui, en 2009 et pour des raisons diverses, ont accordé beaucoup moins de temps à la diffusion nous a semblé moins pertinente. Leur fonctionnement reste néanmoins similaire aux autres compagnies, ce qui tend à confirmer les aspects plus qualitatifs qui seront mentionnés.
} 


\section{Typologie des 572 compagnies selon leur diffusion territoriale en 2009}

\begin{tabular}{|c|c|c|}
\hline \multirow[t]{2}{*}{$\begin{array}{l}\text { "À diffusion importante » lors de l'enquête } \\
\text { réparties en: }\end{array}$} & 503 & $(88 \%)$ \\
\hline & & \\
\hline « régionales » & 103 & $(18 \%)$ \\
\hline «transrégionales » & 259 & $(45 \%)$ \\
\hline «multirégionales» & 95 & $(17 \%)$ \\
\hline « excentrées» & 46 & $(8 \%)$ \\
\hline «À diffusion secondaire » lors de l'enquête & 49 & $(9 \%)$ \\
\hline "Sans diffusion" lors de l'enquête & 20 & $(3 \%)$ \\
\hline
\end{tabular}

* Il s'agit des compagnies qui n'ont pas classé la diffusion de leurs spectacles parmi leurs quatre premières activités en termes d'intensité du temps qui leur est consacré.

ritoriale plus affirmée, sont en proportion plus importants. Ainsi, ils assurent l'essentiel tant de la diversité des propositions artistiques portées par les compagnies professionnelles que de la confrontation de celles-ci avec la diversité des cultures vécues par les personnes. L'importance des établissements non artistiques et des établissements ne disposant pas d'un label national du ministère de la Culture et de la Communication pour le développement des compagnies, et donc plus largement pour l'ensemble du secteur du spectacle vivant, mérite d'être soulignée.

La typlogie établie questionne le modèle de la politique culturelle nationale, qui a construit au cours de la seconde moitié $\mathrm{du} \mathrm{XX}^{\mathrm{e}}$ siècle une hiérarchisation des propositions artistiques en fonction surtout des jugements des experts sur leur qualité.

\section{Activités et ressources des compagnies}

La production des spectacles est la première activité de l'emploi du temps des compagnies. La diffusion et l'administration occupent un rang second, mais d'importance similaire pour ces deux activités. Le temps consacré à la diffusion de spectacles est inversement proportionnel au degré d'inscription territoriale, alors que le temps consacré à l'action culturelle ou à la formation professionnelle croît avec celui-ci : les compagnies régionales sont celles qui consacrent la plus grande partie de leur activité à l'action culturelle et la plus faible à la diffusion, tandis que la relation est inversée pour les compagnies excentrées. Ces dernières organisent plus souvent des manifestations d'action culturelle en relation avec les spectacles, tandis que les compagnies régionales développent fréquemment des dispositifs indépendants de leur offre de spectacles.

$\mathrm{Au}$-delà du primat toujours actuel de la production et de la diffusion de spectacles, l'action culturelle constitue une activité au moins non négligeable - et parfois très importante - des compagnies. Un débat récurrent porte sur le risque d'instrumentalisation politique et sociale des artistes à des fins d'animation des quartiers urbains, d'espaces ruraux et de retissage de liens sociaux. Un grand nombre de metteurs en scène, en piste ou de chorégraphes entendent ainsi se distinguer de l'animation culturelle, qui serait selon eux orientée vers une satisfaction à court terme des besoins de la population. Ils proposent des actions susceptibles
Tableau 1 - Note d'intensité* moyenne des activités principales des compagnies en fonction de leur profil de diffusion

\begin{tabular}{|lccccc|}
\hline & $\begin{array}{c}\text { Production Diffusion } \\
\text { de } \\
\text { Compagnies }\end{array}$ & $\begin{array}{c}\text { Adminis- } \\
\text { spectacles }\end{array}$ & & $\begin{array}{c}\text { Action } \\
\text { tration } \\
\text { culturelle, } \\
\text { formation }\end{array}$ & $\begin{array}{c}\text { Recher- } \\
\text { che }\end{array}$ \\
régionales & 4,0 & 3,0 & 3,1 & 3,2 & 1,3 \\
transrégionales & 4,2 & 3,6 & 3,2 & 2,6 & 1,1 \\
multirégionales & 3,8 & 4,0 & 3,1 & 2,1 & 1,2 \\
excentrées & 4,3 & 4,1 & 2,9 & 1,6 & 1,6 \\
diffusion & & & & & \\
$\quad$ secondaire & 4,2 & 1,0 & 3,3 & 3,3 & 2,4 \\
sans diffusion & 3,3 & 0,3 & 3,6 & 2,6 & 2,5 \\
Ensemble & $\mathbf{4 , 1}$ & $\mathbf{3 , 3}$ & $\mathbf{3 , 2}$ & $\mathbf{2 , 6}$ & $\mathbf{1 , 3}$
\end{tabular}

* La note d'intensité résulte du classement par ordre d'importance des variables considérées dans l'enquête par questionnaire. La note de 5 est attribuée à la $1^{\text {re }}$ activité déclarée par la compagnie, celle de 4 à la seconde et ainsi de suite, ou 0 en cas d'absence d'activité dans un domaine donné.

Source : DEPS, Ministère de la Culture et de la Communication, 2012.

\section{Graphique 1 - Profils de diffusion selon le nombre de représentations médian et moyen en 2009}

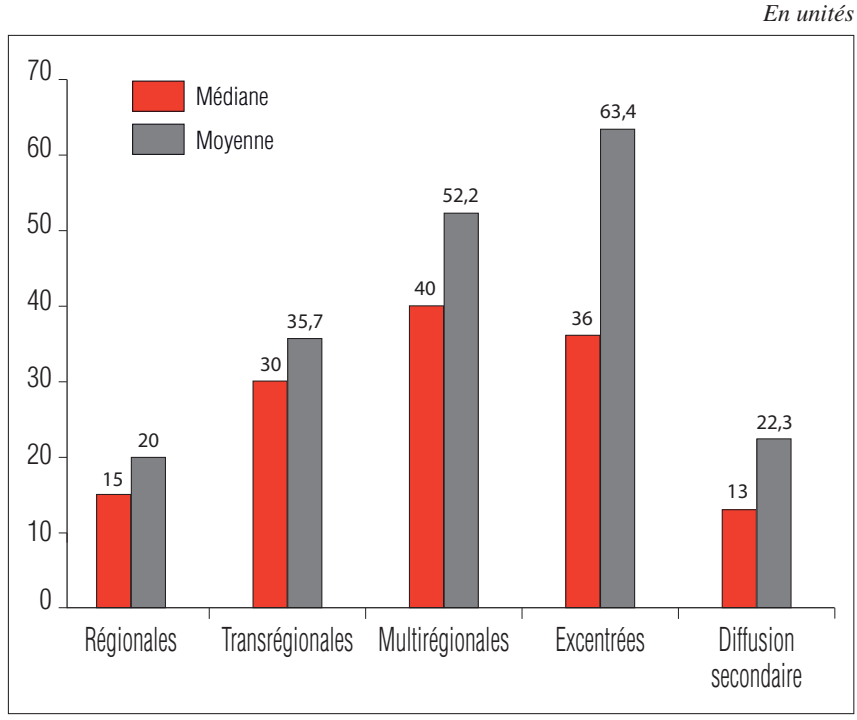

Source : DEPS, Ministère de la Culture et de la Communication, 2012.

d'émanciper les individus par un déplacement de leurs préoccupations vers des mondes sensibles et imaginaires, qui ouvrent symboliquement le champ des possibles. En matière d'action culturelle, la part de l'initiative des compagnies et celle des commandes de collectivités publiques sont maximales pour les troupes régionales, à l'inverse des compagnies excentrées qui ont la part la plus élevée de commandes d'établissements culturels.

L'ancrage régional joue un rôle décisif pour le développement des compagnies, quels que soient les profils territoriaux d'activité.

Tout particulièrement pour les activités de recherche, de production ou d'action culturelle, la région du siège social reste au premier rang dans l'emploi du temps de toutes les compagnies, y compris des excentrées. Ce résultat conforte le sentiment d'un décalage problématique entre la priorité 
Graphique 2 - Note d'intensité moyenne des champs territoriaux dans trois des activités des compagnies

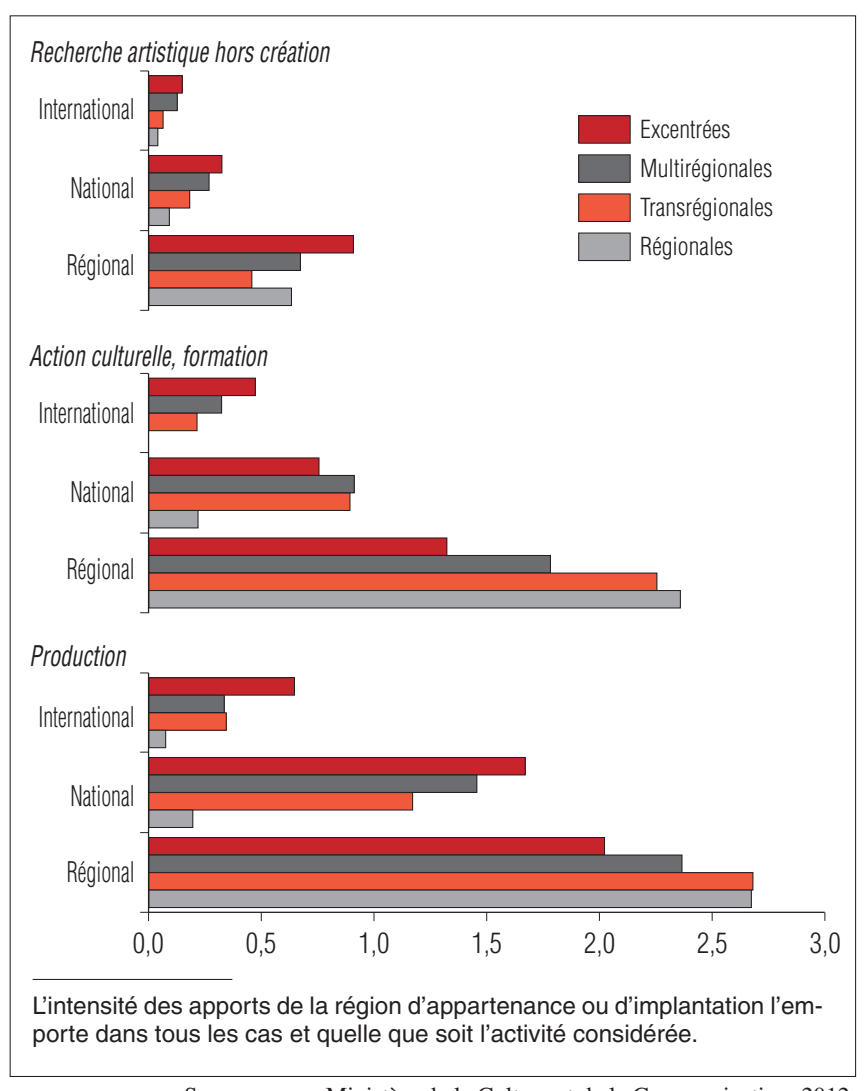

Source : DEPS, Ministère de la Culture et de la Communication, 2012.

institutionnelle donnée à l'excellence artistique sur la territorialité ${ }^{6}$ et la réalité du fonctionnement des compagnies.

Par choix délibéré ou parfois subi au vu des difficultés à diffuser hors de leur région d'origine, les compagnies régionales sont par ailleurs plus orientées vers des relations de proximité avec la population locale. Par là, elles répondent à l'objectif de démocratisation ou de démocratie culturelles. L'élargissement de la zone de diffusion à une échelle nationale et internationale conduit à des formes progressivement moins intensives d'action culturelle, mais se révèle globalement beaucoup plus rémunérateur. Dans l'échantillon qualitatif, le budget moyen des compagnies excentrées est ainsi quatre fois plus élevé que celui des troupes régionales, la part de leurs recettes d'action culturelle ou de formation étant simultanément la plus faible.

$\mathrm{Si}$ les ressources financières des compagnies sont, en moyenne, croissantes depuis les compagnies régionales jusqu'aux compagnies excentrées, on trouve dans chaque groupe des cas dérogatoires, les disparités entre les compagnies étant importantes au sein de chaque catégorie.

Les groupes se différencient d'abord par la structure propre des budgets, selon deux déterminants principaux : la part relative des recettes propres et des subventions publiques d'une part, celle des subventions d'État et des collectivités territoriales d'autre part.

La part des subventions des collectivités territoriales dans le budget est la plus importante pour les compagnies
Tableau 2 - Budget des compagnies selon leur profil de diffusion, 2009

\begin{tabular}{|lrr|}
\hline Compagnies & Moyenne & Médiane \\
régionales & 59100 & 40300 \\
transrégionales & 125000 & 84200 \\
multirégionales & 205200 & 125100 \\
excentrées & 266100 & 137200 \\
diffusion secondaire & 90900 & 56600 \\
sans diffusion & 19800 & 6300 \\
Ensemble & $\mathbf{1 3 1 4 0 0}$ & $\mathbf{7 5 0 0 0}$ \\
\hline
\end{tabular}

Source : DEPS, Ministère de la Culture et de la Communication, 2012.

Tableau 3 - Comparaison de la structure moyenne
des principales ressources
de l'échantillon qualitatif de 2007 à 2009

\begin{tabular}{|c|c|c|c|c|c|}
\hline Compagnies & $\begin{array}{l}\text { Vente } \\
\text { de } \\
\text { spectacles }\end{array}$ & $\begin{array}{l}\text { Copro- } \\
\text { duction }\end{array}$ & $\begin{array}{l}\text { Action } \\
\text { culturelle, } \\
\text { formation }\end{array}$ & $\begin{array}{l}\text { Subven- } \\
\text { tions } \\
\text { d'État }\end{array}$ & $\begin{array}{l}\text { Subven- } \\
\text { tions des } \\
\text { collectivités } \\
\text { territoriales }\end{array}$ \\
\hline régionales & 21,0 & 1,8 & 11,2 & 17,3 & 35,6 \\
\hline transrégionales & 28,5 & 6,4 & 4,1 & 16,7 & 26,5 \\
\hline multirégionales & 41,5 & 7,1 & 5,2 & 14,4 & 19,1 \\
\hline excentrées & 54,9 & 12,2 & 1,2 & 15,7 & 7,1 \\
\hline Ensemble & 37,8 & 7,3 & 4,7 & 15,8 & 20,9 \\
\hline \multicolumn{6}{|c|}{$\begin{array}{l}\text { La partie résiduelle ( } 13,5 \% \text { du budget pour l'ensemble de l'échantillon) cor- } \\
\text { respond aux autres ressources propres comme le remboursement des frais } \\
\text { de tournées, les produits des activités annexes, les subventions civiles, le } \\
\text { mécénat, ou encore à des transferts de charges d'une année à l'autre et au } \\
\text { résultat moyen sur les trois exercices. }\end{array}$} \\
\hline
\end{tabular}

Source : DEPS, Ministère de la Culture et de la Communication, 2012.

régionales, tandis que les recettes propres l'emportent largement pour les compagnies excentrées.

Le chiffre d'affaires - vente de prestations immédiates ou différées (coproductions) à des acheteurs en contrepartie d'une rétribution monétaire - est la première source de revenus des compagnies multirégionales et excentrées, avant les subventions publiques. Cela reflète l'importance des achats de spectacle des établissements artistiques labellisés pour ces troupes. À l'inverse, les subventions publiques constituent la première ressource des compagnies régionales et transrégionales. Il faut souligner également la part supérieure des subventions cumulées des collectivités territoriales par rapport à celles de l'État, sauf pour les compagnies excentrées.

Le rang des revenus de la coproduction est inversement proportionnel à celui des rémunérations tirées de l'action culturelle. Pour les troupes régionales, le rang de la coproduction est ainsi minimal et celui de l'action culturelle et de la formation maximal, tandis que la relation est inversée pour les compagnies excentrées.

Ces modes de financement différenciés révèlent donc des enjeux distincts.

L'échantillon quantitatif donne des indications similaires, bien que moins précises et contrastées. On observe

6. Le rayonnement national et international de la production des établissements culturels labellisés est ainsi placé au premier plan dans les contrats de décentralisation dramatique des CDN. 
Tableau 4-Note moyenne d'intensité des revenus principaux selon le profil de diffusion

\begin{tabular}{|lcccc|}
\hline Compagnies & $\begin{array}{c}\text { Action culturelle, } \\
\text { formation }\end{array}$ & $\begin{array}{c}\text { Copro- } \\
\text { duction }\end{array}$ & $\begin{array}{c}\text { Vente de } \\
\text { spectacles }\end{array}$ & $\begin{array}{c}\text { Subventions } \\
\text { publiques }\end{array}$ \\
régionales & 2,3 & 0,9 & 2,9 & 2,3 \\
transrégionales & 1,8 & 1,1 & 3,3 & 2,7 \\
multirégionales & 1,5 & 1,2 & 3,3 & 2,7 \\
excentrées & 1,1 & 1,7 & 3,4 & 2,1 \\
diffusion second. & 2,1 & 1,0 & 3,0 & 2,4 \\
sans diffusion & 2,0 & 0,6 & 0,0 & 1,6 \\
Moyenne & $\mathbf{1 , 8}$ & $\mathbf{1 , 1}$ & $\mathbf{3 , 1}$ & $\mathbf{2 , 5}$ \\
& & & & \\
"La note d'intensité est ici de 4 (maximale) pour le 1er revenu déclaré par la \\
compagnie et de 1 (minimale) pour le 4e, ou 0 en cas d'absence de revenu \\
dans un domaine donné.
\end{tabular}

Source : DEPS, Ministère de la Culture et de la Communication, 2012.

un primat constant du rang d'importance du chiffre d'affaires sur celui des subventions. Les revenus de l'action culturelle sont toujours supérieurs à ceux de la coproduction, sauf pour les compagnies dites excentrées. La région du siège social constitue une source centrale de revenus pour toutes les troupes, quel que soit leur profil territorial.

La double dépendance économique des compagnies, tant à la vente de leurs prestations à des diffuseurs qu'aux subventions publiques, reste manifeste. La capacité à diffuser les spectacles au-delà de la région d'implantation reste un critère décisif pour le subventionnement étatique et l'entrée en relation avec des établissements labellisés par le ministère, qui sont en mesure de participer plus amplement à la coproduction, à l'offre de résidences et à l'achat de représentations. Il existe donc une tension, actuellement accrue, entre, d'une part, la nécessité ou la volonté d'un plus grand ancrage territorial, et d'autre part, une économie privilégiant une diffusion nomade élargie des propositions artistiques des compagnies.

\section{Des évolutions économiques inquiétantes}

On observe une tendance à la baisse des tarifs unitaires d'achat des spectacles par les diffuseurs et celle, concomitante, des subventions publiques. La moyenne des subventions accordées par les Drac aux compagnies fléchit depuis 2006 (euros constants ${ }^{7}$ ) ; celles délivrées par les collectivités territoriales, dont la progression plus rapide que celles de l'État a permis de représenter, en 2006, les trois quarts des subventions publiques accordées aux réseaux du spectacle vivant en dehors des $\mathrm{Epic}^{8}$, connaissent aujourd'hui au mieux une stabilisation, dans un contexte où la crise économique augmente leurs dépenses sociales et limite l'assiette fiscale des impôts ${ }^{9}$. Dans ces conditions, l'obtention puis la reconduction d'un conventionnement pluriannuel deviennent un enjeu majeur de sécurisation, au moins partielle, d'un avenir aujourd'hui encore plus incertain pour les compagnies.

\section{Graphique 3 - Composition du financement public selon les profils de diffusion des compagnies}

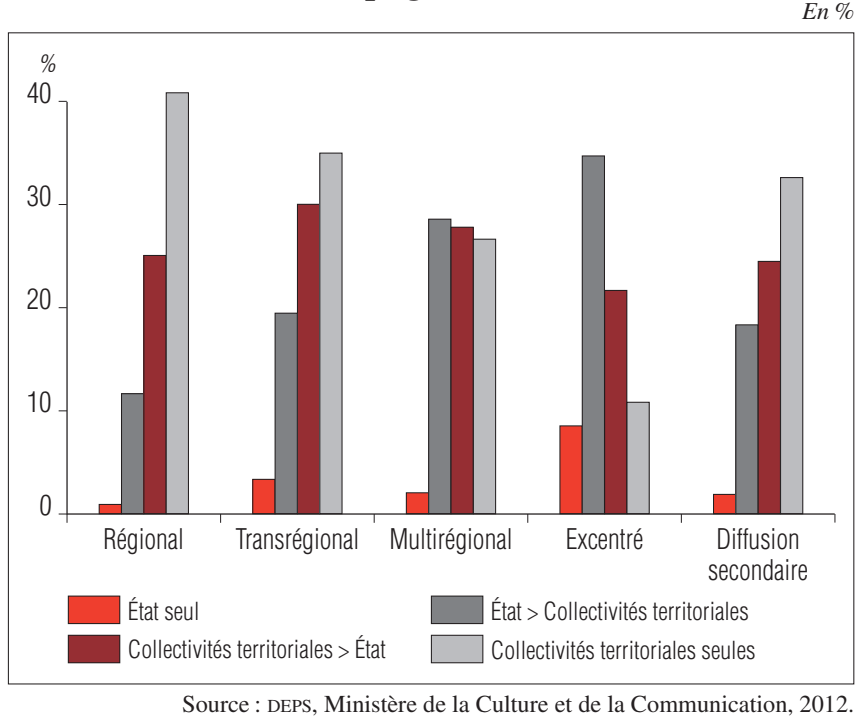

Un phénomène structurel menace l'économie subventionnée des compagnies: les aides publiques progressent moins vite que les coûts fixes des établissements culturels, notamment leur masse salariale, ce qui réduit leur marge pour financer des dépenses artistiques et les contraint à chercher à accroître leurs ressources propres. C'est l'un des facteurs expliquant la baisse du prix d'achat unitaire des prestations par les établissements de diffusion. Les financements européens au profit de plans de développement territorial peuvent offrir un complément de ressources aux compagnies régionales, mais ils nécessitent un accompagnement de la part d'une collectivité locale ou d'un équipement culturel compétent dans la constitution d'un dossier assez complexe. Le mécénat capté par les compagnies est encore très marginal, malgré les avantages fiscaux accrus par la loi du $1^{\mathrm{er}}$ août 2003 et leur élargissement aux dons des particuliers par celle du 25 décembre 2007. Les compagnies chorégraphiques sont celles qui semblent recueillir le plus de dons de la part de fondations, d'entreprises et de ménages.

Les compagnies constatent, dès la fin des années 1990, une détérioration de leurs conditions de diffusion des spectacles, qui s'est encore accentuée ces dernières années. Le mouvement social des intermittents du spectacle en 2003 semble constituer un marqueur historique de cette évolution probablement structurelle.

Face à la faiblesse récurrente des ressources monétaires, les compagnies cherchent à en mobiliser d'autres, non monétaires. En dehors du bénévolat et du travail «invisible », tous deux difficiles à évaluer, le partage de matériel ou d'un local artistique, notamment à l'occasion d'une résidence dans un établissement culturel, est courant.

Les résidences de moyenne ou longue durée dans des établissements artistiques et culturels sont désormais recherchées par les compagnies, pour les ressources monétaires et

7. Chantal LaCroIX, Chiffres clés 2010. Statistiques de la culture, Paris, Ministère de la Culture et de la Communication, DEPS/La Documentation française, 2010, p. 66 et 87 pour les montants des subventions en euros courants; INSEE pour l'indice des prix à la consommation harmonisé.

8. Cartographie nationale du spectacle vivant, Paris, Ministère de la Culture et de la Communication, DMDTs, 2006.

9. La reconfiguration par l'État de la taxe professionnelle a récemment restreint l'assiette fiscale pour le calcul de la contribution économique territoriale. 


\section{Graphique 4-Type de ressource matérielle partagée en fonction du profil de diffusion des compagnies}

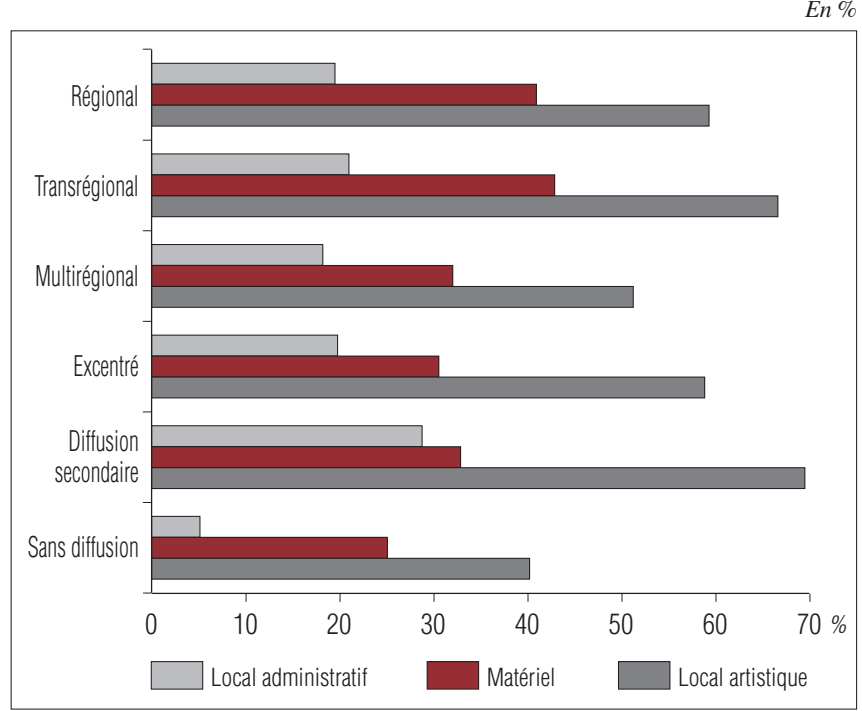

Source : DEPS, Ministère de la Culture et de la Communication, 2012.

non monétaires qu'elles leur apportent et les partenariats pérennes qu'elles instaurent. Cette question est stratégique pour tous les profils de compagnies et renforce, par ailleurs, celle de leur implication territoriale.

\section{Des défis organisationnels accrus}

Sur le plan de l'organisation interne des compagnies, l'échantillon qualitatif confirme que les compagnies sont des micro-organisations flexibles. Chacune est généralement constituée autour du projet artistique d'une ou deux personnes, même si quelques cas de collectifs, d'organisations familiales ou de compagnies constitués par des amis sont aussi repérables. La compagnie intègre, dès que possible, au moins une personne plus spécifiquement chargée des tâches d'administration et, plus largement, de la production et de la diffusion, en particulier de spectacles. Des artistes et techniciens (entre 5 et 10 la plupart du temps) participent régulièrement au développement du projet d'ensemble, tout en poursuivant des collaborations avec d'autres compagnies ou organisations artistiques et culturelles. Le fonctionnement repose donc d'abord sur un agencement variable de moyens, selon les besoins immédiats ou de court terme. Élément central de la spécificité et de la qualité artistiques des compagnies, la capacité à développer un partenariat de long terme avec une équipe artistique et technique ne paraît donc ni aisée ni constante. Ce mode de fonctionnement fait de chaque compagnie une véritable « entreprise-réseau », qui utilise une gamme variée de ressources et de compétences, disponibles dans l'environnement professionnel et pouvant relever d'une diversité d'organisations. Cette dynamique est également facilitée par les possibilités réglementaires de flexibilité d'emploi accordées aux arts vivants, notamment avec le recours intensif des compa-

\section{Graphique 5 - Nombre moyen de personnes engagées selon le type de contrat pour les différents profils de diffusion}

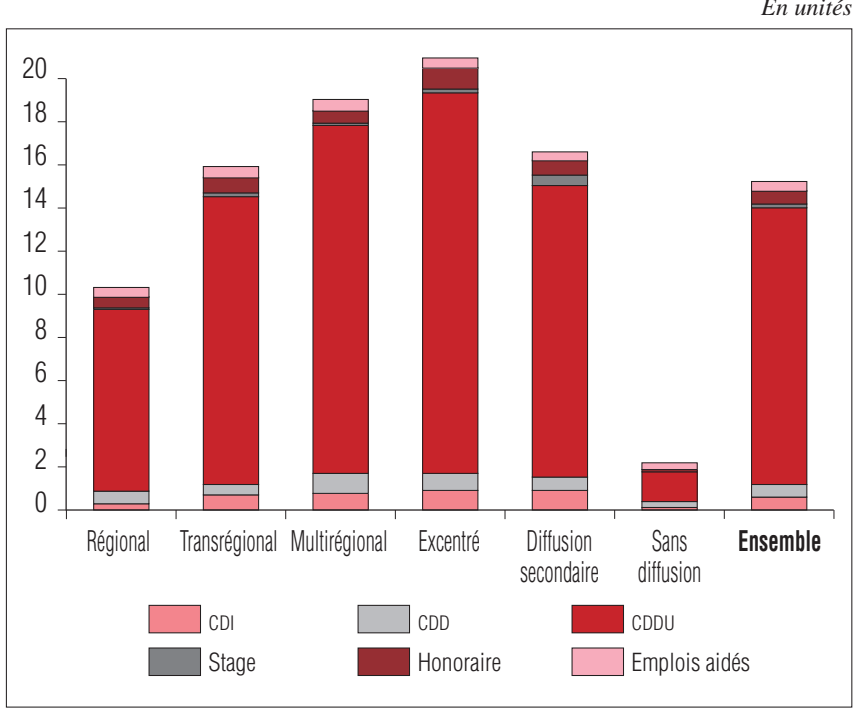

Source : DEPS, Ministère de la Culture et de la Communication, 2012.

gnies au contrat à durée déterminée d'usage (CDDU), qui peut représenter jusqu'à $90 \%$ ou plus de leur masse salariale. Aujourd'hui, les compagnies s'organisent structurellement toujours à partir et autour de l'emploi intermittent ${ }^{10}$.

Le croisement des données statistiques collectées et celles de l'Unedic indique que les indemnités de chômage des intermittents du spectacle représentent, pour les compagnies, un complément économique estimé autour de $20 \%$ par rapport à leur budget.

En termes de gouvernance, les compagnies ont toujours très majoritairement recours au statut d'association à but non lucratif. Dans ce cadre, les conseils d'administration, très souvent réduits à un bureau de trois ou quatre membres, n'ont la plupart du temps pas de poids réel dans la définition des orientations et la marche courante des compagnies. Néanmoins, et dans le cadre d'un environnement complexifié pour les compagnies, le souci est perceptible de s'appuyer de plus en plus sur les membres de ces bureaux pour des apports en conseil et en compétence. Le statut associatif ne conduit donc pas à une mise en cohérence évidente entre, d'une part, le pouvoir légal qui revient de droit au président, et d'autre part, la responsabilité sociale et fiscale si le détenteur de la licence d'entrepreneur de spectacles est une personne différente, et, enfin, la direction artistique.

La moitié des compagnies de l'échantillon qualitatif mentionne un binôme véritablement opérant entre la direction artistique et une personne qui porte une forte responsabilité d'administration de la compagnie. Mais, si une collaboration de long terme avec un même administrateur existe, elle reste l'exception. Ainsi, le problème de la rotation rapide des personnes en charge de tâches d'administration est mentionné, d'autant qu'une bonne part de ces postes dépend d'emplois aidés, par définition temporaires.

10. Sur cet aspect comme sur de nombreux autres, la situation actuelle des compagnies ne diffère pas fondamentalement de ce qui était repérable dès la fin du siècle dernier. Voir par exemple, Philippe HenRY, «Les compagnies théâtrales, une pluralité de logiques à articuler », Théâtre/Public n 153 , maijuin 2000, p. 67-74 et «Compagnies théâtrales : les particularités d'un vrai jeu d'Arlequin », Théâtre/Public n 168, mai-juin 2003, p. 4-22. En revanche, les conditions contextuelles ont continué à changer en dix ans et agissent de plus en plus sur les orientations et le fonctionnement interne des compagnies. 


\section{Graphique 6-Répartition des compagnies selon le détenteur de la (des) licence(s) d'entrepreneur de spectacles en fonction de la discipline artistique}

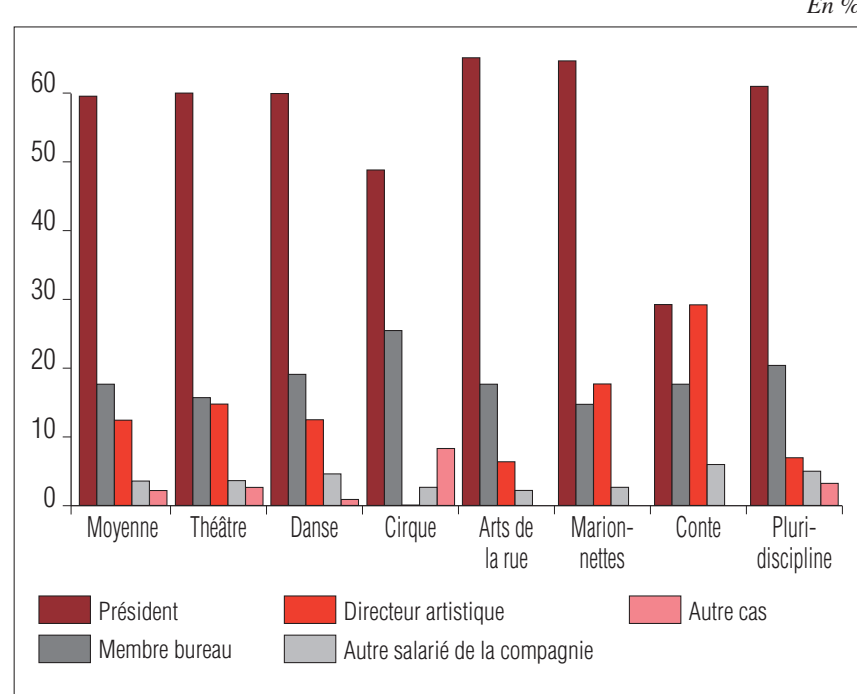

Source : DEPS, Ministère de la Culture et de la Communication, 2012.

\section{Graphique 7 - Recours des compagnies aux différentes formes d'agence privée d'accompagnement}

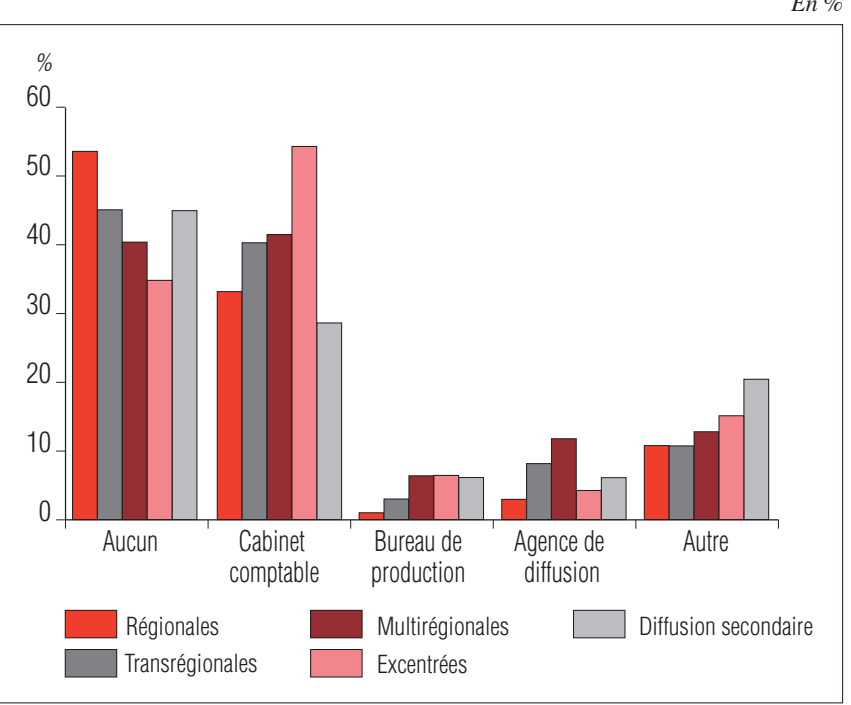

Source : DEPS, Ministère de la Culture et de la Communication, 2012.

Par ailleurs, le recours à l'externalisation des tâches administratives se développe, sous la forme, le plus souvent, de sous-traitance de la comptabilité et de la paye. L'appel à des savoir-faire extérieurs (dont la collaboration avec un bureau de production ou une agence privée de diffusion) croît depuis les compagnies régionales jusqu'aux excentrées. Sur un autre plan, les compagnies dirigées par une femme sont plus souvent représentées dans les compagnies régionales et, de manière plus attendue, dans les compagnies chorégraphiques, mais aussi dans celles dont les budgets sont les moins importants. Si l'interprétation de ces éléments ne doit pas être univoque, ils indiquent au moins une situation différenciée entre les compagnies dirigées par une femme et celles qui le sont par un homme.

\section{L'impact de la crise actuelle}

Certains éléments de la crise économique de 2008-2009 sont perceptibles. Paradoxalement, le budget moyen des compagnies de l'échantillon qualitatif a augmenté en 2009. Ce phénomène s'explique d'abord par une hausse des aides publiques, notamment de la part des conseils généraux et des ministères autres que celui de la Culture et de la Communication, ainsi que par un nombre plus important de représentations. L'impact négatif de la récession économique s'est surtout manifesté par une baisse du prix unitaire des spectacles, signalée dans les entretiens et estimée à $40 \%$ en deux ans d'après l'analyse des comptes des compagnies. D'après l'évolution de la masse salariale artistique et technique par représentation, la moitié de cette baisse est imputable à une réduction de la taille moyenne des spectacles, en lien avec une demande accrue de « formes légères », l'autre moitié à un effet de la concurrence par les prix.

Ces divers éléments montrent, de fait, un accroissement de la production de spectacles, dans des conditions de plus grande concurrence à la diffusion, même si la crise a affecté différemment les compagnies, selon leur profil de diffusion et la nature de leurs relations partenariales. Les réponses relatives aux perspectives d'évolution des ressources reflètent globalement la fragilité économique de la très grande majorité des compagnies. $27 \%$ des compagnies de l'échantillon quantitatif déclarent une perception pessimiste ou inquiète, contre $14 \%$ se déclarant optimistes. La position majoritaire $(59 \%)$ relève d'une posture prospective, d'abord focalisée sur les moyens envisagés pour accroître les ressources propres et les aides publiques.

Le diagnostic d'une situation socio-économique d'ensemble particulièrement problématique pour l'avenir, à mode structurel de fonctionnement et de régulation inchangé, peut donc être établi. Pour l'instant, chaque compagnie reste incitée à poursuivre un développement singularisé, d'abord centré sur le renouvellement constant de spectacles pouvant être largement diffusés au-delà de leur

\section{Graphique 8-Perception des compagnies quant au devenir de leurs ressources}

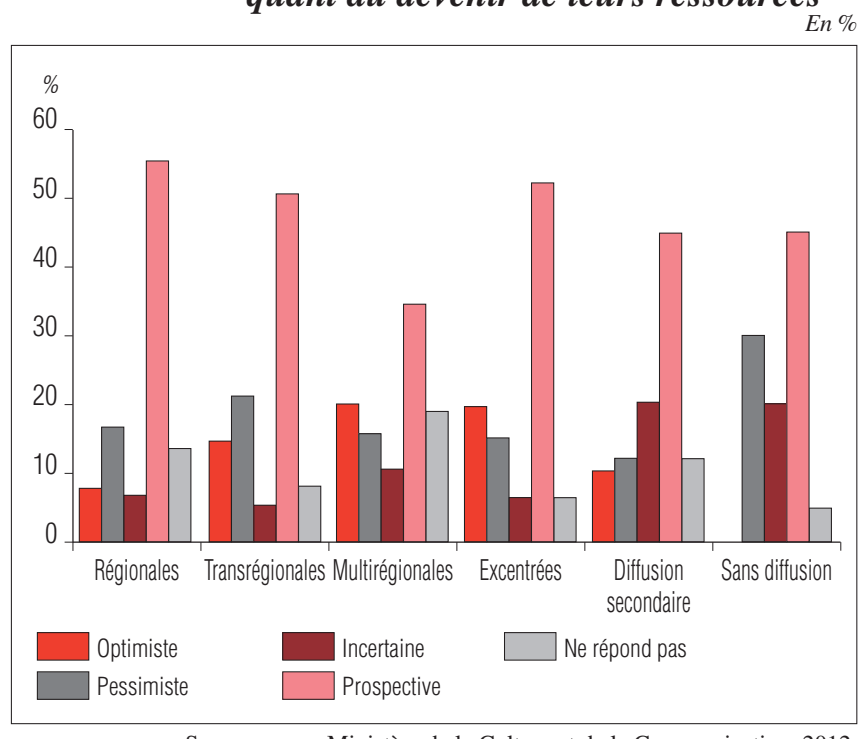

Source : DEPS, Ministère de la Culture et de la Communication, 2012. 
région de création. Le risque est d'accroître la tension entre une multiplicité d'œuvres cherchant des lieux de programmation et des débouchés sur un marché dont les capacités de développement ne sont pas à la mesure de cette offre toujours plus abondante.

\section{Des compagnies partie prenante d'une filière spécifique}

L'étude permet d'appréhender l'état actuel de structuration de la filière des arts théâtraux et chorégraphiques et la situation des compagnies dans ce contexte spécifique. L'approche par la notion de filière revient en effet à considérer les compagnies en France comme acteurs décisifs d'un ensemble d'entreprises et d'organisations interdépendantes visant à assurer un processus global, allant de la conception d'un type de biens ou de services à sa fabrication et à sa mise sur un marché, jusqu'à son appropriation par des usagers. Dans ce processus, qui va d'un amont (conception et production) jusqu'à un aval (diffusion et appropriation), chaque organisation particulière intervient de manière partielle et localisée. Pour le spectacle vivant, le genre de biens et de services à considérer comprend aujourd'hui, non seulement les spectacles comme œuvres, mais aussi les pratiques d'action artistique et culturelle qui leur sont associées.

Sur ce plan, il faut souligner l'impossibilité des compagnies à pouvoir, de nos jours, se contenter d'une centration sur la double fonction de production et de diffusion de spectacles. Ce dipôle reste la référence centrale et constamment revendiquée des directeurs, en particulier artistiques, interrogés. Dans les faits cependant, on observe que chaque compagnie doit, par intérêt propre et par nécessité dictée par l'environnement, se préoccuper toujours plus d'autres fonctions vitales dans le fonctionnement de la filière du spectacle vivant. Cinq grandes fonctions sont identifiées.

Les compagnies sont d'abord confrontées à la très forte incertitude de la fonction de recherche-expérimentation. Elles sont contraintes d'y faire face en mobilisant surtout une économie réticulaire et de réciprocité, finalement assez peu collectivement réfléchie et organisée. Sur ce plan, le régime partenarial entre privé et public qui s'est structuré dans la seconde moitié du $\mathrm{XX}^{\mathrm{e}}$ siècle a abouti à des dispositifs partiels et fragmentés d'aide à la production, sans prise en compte globale des coûts et sans régulation territoriale (locale ou nationale) du segment recherche-expérimentation. Celui-ci est pourtant de plus en plus central dans l'économie créative contemporaine, dont les mondes de l'art participent. Face au peu de ressources dont elles disposent à cet égard, les compagnies optent le plus souvent pour des formules d'atelier ou de laboratoire de recherche, où la mise à disposition réciproque de moyens matériels (dont des locaux) et de compétences artistiques ou autres est la règle.

La fonction de production-fabrication est mieux appréhendée et a été l'un des piliers du mode de développement de l'art dont nous héritons. Mais pour les arts théâtraux et chorégraphiques, elle continue à être principalement envi- sagée dans des partenariats locaux et successifs entre les compagnies, les établissements artistiques et culturels étant sollicités comme coproducteurs, les pouvoirs publics attribuant des subventions. Ces divers soutiens sont encore largement conçus au sens restreint d'une aide à la production, sans que les fonctions (et les coûts associés) de distribution ou de diffusion (et la question d'un retour sur investissement en cas de large diffusion) soient réellement intégrées.

La fonction de distribution-médiatisation est elle aussi très peu réfléchie et organisée collectivement pour les arts théâtraux et chorégraphiques. Le fait que le Festival off d'Avignon - essentiel pour les compagnies comme premier «salon professionnel » de France - ne fasse toujours pas l'objet d'une prise en charge plus collective de la part de l'ensemble du milieu professionnel et des pouvoirs publics en est un exemple frappant.

Dans ces conditions et avec les moyens matériels, humains et financiers dont ils disposent, les établissements artistiques et culturels sont toujours plus au centre des décisions, tant pour les trois fonctions précédentes que pour celle de diffusion-exploitation qui organise les conditions concrètes selon lesquelles les spectateurs et autres praticiens amateurs feront l'expérience des propositions artistiques des compagnies. Ils constituent d'ailleurs l'autre pilier essentiel du mode de développement de l'art hérité de la seconde moitié du $\mathrm{XX}^{\mathrm{e}}$ siècle. Pourtant, ce sont surtout les établissements non labellisés par le ministère de la Culture et de la Communication qui se distinguent par le nombre et par la variété des propositions théâtrales ou chorégraphiques produites et diffusées ${ }^{11}$, en dépit d'une faiblesse persistante de moyens consacrés aux propositions d'action culturelle, dans tous les segments de la filière, et de la très inquiétante tendance à la baisse des tarifs unitaires d'achat de spectacles.

Enfin, si la fonction de réception-appropriation était plus difficile à appréhender, il se confirme que les compagnies sont largement impliquées dans ce qui s'apparente à la proposition de véritables services relationnels, au-delà de la seule production et diffusion de spectacles. Reste que la prise en compte, au cœur même de leur projet artistique, de la diversité culturelle et générationnelle de nos concitoyens et des territoires dans lesquels ils vivent n'est perceptible que pour un nombre restreint de compagnies. Un des enjeux sous-jacents aux mutations générales de notre société consiste pourtant dans l'invention de relations plus symétriques et plus interactives entre professionnels et amateurs d'art, ou simplement curieux de propositions artistiques qui tiendraient compte de leur propre culture vécue.

Les compagnies théâtrales et chorégraphiques apparaissent donc comme autant de micro-entreprises flexibles, construisant et entretenant entre elles et avec leurs différents partenaires professionnalisés de très nombreuses relations. Celles-ci sont déjà indispensables pour assurer l'émergence des compagnies et toujours plus nécessaires pour permettre leur développement.

Les compagnies doivent désormais développer un grand nombre de compétences et mobiliser par elles-mêmes des savoir-faire spécifiques à chacune des fonctions de la filière.

11. Dans l'échantillon qualitatif, ces établissements ont représenté en moyenne $65 \%$ de la diffusion des compagnies entre 2007 et 2009. 
Elles n'en ont pourtant pas vraiment les moyens, alors même qu'une articulation un peu plus systématique de l'ensemble des acteurs impliqués reste encore largement à concevoir et mettre en œuvre. Le partenariat entre privé et public qui a permis l'expansion et la structuration du monde du spectacle vivant depuis l'après Seconde Guerre mondiale $^{12}$ demeure décisif, tout en rencontrant désormais de nombreuses limites. Pour autant, ni la proposition ni l'expérimentation de nouvelles façons de s'y prendre, en particulier plus collectivement, n'émergent vraiment. Il s'agit là d'un point nodal particulièrement problématique pour les années à venir.

Quoi qu'il en soit, il faut souligner un phénomène essentiel dans le contexte actuel : l'émergence et le développement de chaque compagnie renvoient au premier chef à sa capacité à se rendre visible dans la filière et, en particulier, à être sélectionnée par divers canaux de valorisation symbolique et de redistribution de fonds, dont les plus pérennes restent les conventionnements pluriannuels, essentiellement négociables avec des centres culturels et les différents pouvoirs publics dont ils dépendent et qui ont leurs propres enjeux et territorialités d'action.

\section{REPENSER LE MODE DE DÉVELOPPEMENT DES COMPAGNIES ?}

\section{Une remise en cause du modèle de service public culturel}

L'analyse de l'état actuel de la filière du spectacle vivant et des mutations, internes et contextuelles, dans lesquelles elle se trouve engagée implique une mise à distance critique de son mode de développement historique, même s'il a eu l'immense avantage de permettre une croissance et une vitalité de ce secteur d'activité artistique.

Sur un plan plus strictement économique et organisationnel, l'analyse de la filière du spectacle vivant doit prendre en compte l'hybridation croissante des logiques de marché et des logiques de service public, mais également l'importance désormais structurelle d'un tiers secteur, d'initiative privée mais producteur de biens et de services selon des buts non lucratifs. La croissance continue de ce tiers secteur depuis les années 1980 montre la nécessité de mobiliser un troisième ensemble de logiques portant sur les échanges en réciprocité et réticulaires (bénévolat, mises à disposition à titre gratuit de moyens ou de compétences, relations interpersonnelles dans des réseaux...). Si presque toutes les compagnies se reconnaissent dans ce modèle de référence, l'utilisation exclusive du statut associatif n'induit pourtant pas nécessairement des options de développement plus mutualistes ou solidaires, qui ne concernent encore qu'une faible minorité de compagnies. Cette probléma- tique d'économie «ternaire » est de plus en plus prégnante dans les organisations relevant de l'économie sociale et solidaire. Elle mériterait d'être mieux déclinée et spécifiée pour le spectacle vivant et plus particulièrement pour les compagnies théâtrales et chorégraphiques. Celles-ci, de plus en plus nombreuses, sont en effet confrontées à la difficulté de maîtriser la complexification des différentes phases, étroitement imbriquées et risquées, de leur filière d'activité. La stratification des compagnies, dans leur accès aux ressources, monétaires ou non, repose toujours en grande partie sur les relations partenariales qu'elles arrivent à établir et à stabiliser avec deux autres types d'acteurs décisifs : les établissements artistiques ou culturels, directement intéressés par les prestations possibles des compagnies, et les collectivités publiques, plus préoccupées de leurs apports au titre de l'intérêt collectif ou général.

De plus, le phénomène de concentration en aval de la filière d'une grande partie de la valorisation symbolique et économique, au moment de la distribution, de la diffusion et de l'appropriation des spectacles, crée un rapport d'échange inégal entre les établissements artistiques ou culturels et les compagnies. Ces micro-entreprises assument en effet la prise de risque principale dans les phases de recherche-expérimentation et de production-fabrication, dans un contexte où l'intensification de la concurrence accroît l'incertitude des jugements sur la qualité qui seront portés par les divers prescripteurs, privés et publics, et par les publics eux-mêmes. Le régime de l'assurance-chômage des intermittents et les échanges fondés sur la réciprocité constituent les principales ressources «invisibles » pour faire face à cette situation. Un conventionnement pluriannuel par des collectivités publiques, ainsi que des résidences de longue durée dans des établissements artistiques ou culturels sont susceptibles de stabiliser l'horizon de production et de diffusion pour la minorité des troupes qui en bénéficient.

Le contexte concurrentiel, qui se manifeste par une baisse du prix unitaire des représentations, constitue également une force de désintégration des liens de coopération dans l'ensemble du spectacle vivant et plus particulièrement pour les compagnies. Elle s'inscrit dans les transformations contemporaines de la filière du spectacle vivant. Les compagnies critiquent particulièrement la tendance à une industrialisation de leurs rapports avec les établissements artistiques ou culturels, sous la forme d'une uniformisation des programmations et d'une marchandisation de la diffusion dans leurs rapports au public. Le fonctionnement actuel de la filière et les divers changements qui s'y opèrent conduisent ainsi à un très fort sentiment de remise en cause du modèle historique du service public culturel, essentiellement fondé sur l'articulation entre les exigences artistiques et la démocratisation culturelle. Sur ces questions jusqu'à présent peu prises en compte, il convient de ne pas dissocier l'approche en termes de valeurs et d'objectifs d'intérêt

12. Partenariat dont ont d'abord bénéficié les établissements artistiques labellisés et les artistes de grande notoriété, mais aussi la pluralité des compagnies et plus largement l'ensemble des organisations du spectacle vivant professionnel. Il a également abouti à une gestion très morcelée de ce secteur d'activité, la multiplication des « guichets » financiers auprès desquels chaque projet tente de réunir les ressources qui lui sont nécessaires se croisant avec la stratification hiérarchisée actuelle de ce monde de l'art. Dans le même temps, un nombre toujours croissant de compagnies et de praticiens voulant se professionnaliser est confronté à des difficultés d'autant plus renforcées que la conception tant de la diffusion des spectacles que de l'appropriation de ceux-ci par des non-professionnels a finalement fort peu évolué structurellement. 
général et celle s'attachant à promouvoir des dispositifs organisationnels et des modalités de régulation qui apparaissent aujourd'hui pertinents.

De ce point de vue, mieux comprendre la situation des lieux de production et de diffusion pluridisciplinaires ne disposant pas de label national délivré par le ministère de la Culture et de la Communication serait essentiel. Ces lieux jouent un rôle de premier plan, tant pour les compagnies que, plus globalement, pour la présence démocratisée et diversifiée des arts vivants sur l'ensemble du territoire national. Une étude récente sur la région Île-de-France, où sont implantées près d'un tiers des compagnies de spectacle vivant, permet d'insister sur des éléments désormais déterminants, tout en recoupant et validant nos propres résul$\operatorname{tats}^{13}$.

\section{Lieux de diffusion pluridisciplinaires de spectacle vivant en Île-de-France majoritairement financés par les communes et intercommunalités, Opale/Arcadi, juillet 2011}

Sur l'ensemble de la région francilienne, l'étude recense plus de 200 lieux de diffusion pluridisciplinaires principalement financés par les municipalités et leurs regroupements intercommunaux. Elle repère également près de 300 lieux spécialisés dans une discipline (musiques actuelles, par exemple) ou gérés par une compagnie, ainsi que plus de 200 autres lieux (espaces socioculturels, lieux indépendants pluridisciplinaires comme les friches culturelles). Soit un total de plus de 700 lieux non labellisés par le ministère de la Culture et de la Communication pour un peu plus de 50 lieux labellisés en 2011.

Sur un échantillon d'une centaine de lieux de diffusion pluridisciplinaires où ces données étaient disponibles, l'étude de la programmation de la saison 2009-2010 révèle que le nombre de spectacles programmés est 10 fois plus élevé que dans les neuf scènes nationales de la région. En dépit de séries de représentations plus courtes (une moyenne inférieure à 2 représentations par spectacle programmé, quelle que soit la discipline artistique considérée), ces lieux de diffusion pluridisciplinaires non labellisés ont touché 5 fois plus de spectateurs que les scènes nationales de la région, avec 5,5 fois plus de représentations. Si on prolonge les moyennes à la totalité de ces lieux, les ratios donnent 16 fois plus de spectacles programmés, 8,5 fois plus de représentations, et 8 fois plus de spectateurs touchés que pour les neuf scènes nationales franciliennes.

La concentration est extrêmement forte : sur plus de 2000 spectacles distincts programmés par la centaine de lieux plus particulièrement étudiés, seuls 2,1\% l'ont été plus de 10 fois (et $0,6 \%$ plus de 15 fois). De même, seuls 2,3\% de ces spectacles ont été accueillis dans plus de cinq lieux de l'échantillon (et $0,2 \%$ dans plus de 10 lieux).

Ces établissements ont une marge financière à consacrer aux dépenses artistiques qui correspond à $30 \%$ de leur budget, et dans les faits, est inférieure puisque la mise à disposition du personnel municipal n'est pas évaluée dans de nombreux budgets. Entre une professionnalisation accrue des directions de ces établissements depuis les années 1990, mais aussi une plus grande proximité avec les élus décideurs et les directeurs des affaires culturelles (Dac) en charge de la mise en œuvre de la politique culturelle locale, la gouvernance de ces lieux s'est nettement complexifiée.
L'étude réalisée à un niveau régional confirme que les lieux non labellisés par le ministère assurent la plus grande part de la diffusion des spectacles proposés par les compagnies. Elle met également en exergue la concentration de la diffusion sur quelques spectacles et, partant, les très fortes inégalités selon les œuvres proposées et les compagnies considérées. Ces établissements disposent pourtant d'une faible capacité pour financer les dépenses artistiques et sont également soumis à une pression de leurs tutelles publiques pour dégager des ressources propres élargies, tout comme les établissements culturels labellisés.

On voit aussi se renforcer la place donnée à l'action culturelle et aux pratiques artistiques en amateur, à proportion de l'identité vécue de «théâtres de proximité » et de l'intérêt porté à une plus grande participation de la population locale à la vie de ces lieux. Avec des équipes permanentes souvent réduites, l'appel à des compagnies et pour des résidences plus ou moins longues dans le lieu constitue la stratégie la plus employée.

Au final, ces lieux de programmation jouent un rôle désormais irremplaçable, tant pour la diffusion du spectacle vivant que pour l'action culturelle, l'accueil des compagnies et donc également pour la production de spectacles.

Il existe donc des relations interorganisationnelles renforcées et toujours plus déterminantes entre compagnies et établissements artistiques ou culturels territorialisés. Plus globalement, le devenir des interactions entre les compagnies, les établissements artistiques et culturels (avec ou sans label national), les pouvoirs publics et les publics euxmêmes soulève des interrogations systémiques sur l'organisation présente et future de la filière du spectacle vivant.

\section{Entre ancrage territorial et nomadisme dans des réseaux}

Micro-entreprises flexibles, construisant et entretenant entre elles et avec leurs différents partenaires professionnalisés de très nombreuses relations, les compagnies théâtrales et chorégraphiques doivent développer un plus grand nombre de savoir-faire en relation avec les différentes fonctions constitutives de leur filière d'activité. Les compagnies peinent à s'adapter à cette situation et à mettre en œuvre des modes d'action plus collectifs. Certaines expérimentations émergent, mais restent encore très locales et largement en deçà de l'enjeu général.

Aujourd'hui, la contradiction socio-économique majeure de toute compagnie est donc d'être une organisation-réseau, devant impérativement construire une permanence entrepreneuriale, tout en restant constamment hyperflexible. Elle doit simultanément se comporter comme une entreprise totale, c'est-à-dire qui assume la pluralité des tâches indispensables pour couvrir le processus complet du spectacle vivant, depuis l'émergence d'une idée de proposition artistique jusqu'à son appropriation physiquement éprouvée par divers publics.

Dans un contexte d'incertitude et de concurrence accrues, chaque compagnie va être de plus en recherche de

13. OpALE, Lieux de diffusion pluridisciplinaires de spectacle vivant en Île-de-France majoritairement financés par les communes et intercommunalités, Paris, Arcadi, juillet 2011. 
résidences de durée conséquente (de un à trois ans, voire plus) sur un territoire donné et en association avec au moins une organisation artistique et culturelle déjà reconnue, localement et au sein de la filière des arts vivants. Mais plus largement, construire une notoriété au plan local demande de développer dans la durée des activités et des partenariats avec une diversité plus ou moins grande d'acteurs sociaux et d'organisations, dont les préoccupations centrales ne sont pas nécessairement artistiques. On passe alors de la nécessité artistique et socio-économique des résidences aux enjeux culturels plus complexes et aux conditions de réussite plus délicates d'une implantation. La tendance repérée à une plus grande territorialisation de l'activité des compagnies renvoie donc autant à la volonté d'un ancrage de proximité des échanges relationnels et partenariaux qu'à la nécessité d'une diversification de leurs ressources.

Simultanément, des partenariats avec des artistes, des équipes ou des organisations situés en dehors du territoire privilégié d'activité favorisent l'ouverture à une variété d'expériences artistiques et de contextes culturels. Ces partenariats se construisent d'abord au fil d'opportunités particulières et de rencontres personnelles. Ils constituent peu à peu le tissu réticulaire qui est au fondement des possibilités de survie et de développement de chaque compagnie. Cette dynamique joue un rôle majeur, par exemple dans la capacité de diffusion des spectacles et des autres propositions artistiques et culturelles d'une compagnie, dans et au-delà de sa région d'appartenance, comme dans la recherche de nouveaux lieux de résidence, temporaires ou de plus longue durée. On comprend alors le rôle essentiel des partenariats fidélisés, tant auprès d'organisations artistiques et culturelles que des instances publiques ou des sociétés civiles attentives aux propositions renouvelées des compagnies.

L'enjeu est d'articuler un ancrage territorial, comme base de production, avec un élargissement du réseau de diffusion au-delà de la région d'implantation afin de gagner en visibilité auprès des professionnels et des médias. Certains directeurs artistiques expriment aussi la nécessité de se ressourcer au contact de la vie culturelle et artistique d'autres régions ou pays, afin d'entretenir un sens de la créativité et un sens de la rencontre toujours plus aiguisés.

\section{Mieux accompagner les compagnies?}

Comment accompagner les compagnies dans les différentes phases de leur activité ? La coopération interorganisationnelle dans le sens d'une économie solidaire est une perspective envisagée par une minorité d'entre elles. Elle se heurte aux fortes pressions concurrentielles, qui tendent à distendre les rapports de collaboration et à accentuer la diversification des trajectoires de reconnaissance professionnelle.

Dans ce contexte, l'externalisation de tâches administratives dans des bureaux de production offre l'avantage d'une division du travail a priori plus opérationnelle. Elle permet au noyau central de la compagnie de se recentrer sur le cœur de son identité artistique et de bénéficier de compétences spécialisées, partagées avec d'autres troupes, pour les aider dans les phases de production, de distribution et de diffusion. Elle nécessite néanmoins la construction d'une relation de confiance, afin que les objectifs artistiques et culturels de la compagnie soient respectés. Cette opportunité peut se heurter à un problème de financement, notamment pour les compagnies émergentes, la tarification des prestations devant être à la fois supportable pour la troupe et viable pour le bureau.

La production déléguée offre également aux compagnies un cadre très utile de mise à disposition de compétences administratives d'un établissement culturel ou, là encore, d'un bureau de production. Elle suppose de bien s'entendre sur les marges de manœuvre de la direction artistique des compagnies aidées, en fonction de la contrainte budgétaire du lieu ou du bureau d'accueil.

Les conventions de compagnonnage artistique, expérimentées par exemple dans les arts de la marionnette, sont également susceptibles de permettre un parrainage de jeunes artistes par des troupes plus expérimentées.

La question d'une plus grande permanence des emplois dans les compagnies est également cruciale pour permettre leur développement dans un contexte d'hyperflexibilisation des relations de travail. Si les emplois artistiques et techniques restent structurellement dépendants des formes d'emploi intermittentes, les aides publiques à l'emploi constituent une forme très utile pour amorcer la stabilisation d'un noyau administratif. Elles devraient au moins être adaptées et étendues aux directeurs artistiques, pivots essentiels mais très souvent en situation de grande fragilité économique.

D'abord centré sur les projets portés par des établissements à fortes notoriété et visibilité sociale, le mécénat ne peut raisonnablement constituer une ressource structurelle pour les compagnies. Dans le meilleur des cas, il ne constitue aujourd'hui qu'une force d'appoint pour financer des projets sans doute plus éducatifs et sociaux qu'essentiellement artistiques.

Enfin, si les relations entre les établissements artistiques ou culturels et les compagnies se sont plus tendues au début $\mathrm{du} \mathrm{XXI}{ }^{\mathrm{e}}$ siècle, elles dépendent aussi largement des orientations des politiques culturelles.

\section{Les scénarios ministériels prospectifs}

Des quatre scénarios sur le devenir des politiques culturelles à l'horizon 2030 : l' « exception continuée », le «marché culturel », l' « impératif créatif », la «culture d'identités ${ }^{14} »$, seul le dernier scénario prend en compte la question de la diversité des cultures vécues par nos compatriotes et celle, associée, de la pluralité des propositions artistiques mises en œuvre, par exemple, par les compagnies. Il met en scène une segmentation de l'intervention publique entre un État modeste, qui appuierait les fleurons artistiques de la nation, et des collectivités territoriales qui soutiendraient un « art social ». L'hypothèse centrale de cette vision repose sur

14. Culture \& Médias 2030. Prospective de politiques culturelles, Paris, Ministère de la Culture et de la Communication, DEPS, coll. «Questions de culture», 2011. 
la poursuite de la crise économique, qui provoquerait un retour au protectionnisme, une exacerbation des conflits sociaux et une pluralisation des identités locales ou communautaires, plutôt repliées sur elles-mêmes. Le courant de l'art social, destiné à « développer la sensibilité et le sens critique collectifs », et donc l'ouverture aux autres, ne pourrait alors se développer que dans un contexte de recul des financements publics et d'écart croissant entre, d'une part, la culture multimédiatique et populaire, d'autre part, la culture artistique légitime.

La pluralité et la complémentarité des troupes selon leur ancrage territorial entrent plus particulièrement en résonance avec ce scénario. Mais elles nous paraissent poser plutôt la question d'un rééquilibrage général des aides publiques, en faveur de la diversité non seulement de l'offre artistique et culturelle, mais aussi de ses modes d'appropriation par les publics. Cela pourrait conduire à un cinquième scénario, qui n'est cependant économiquement viable et politiquement soutenable que si les pratiques culturelles de la population trouvent leur propre compte dans cette diversification de l'offre. Cela renvoie à des questions qui ne font pas aujourd'hui l'objet de consensus dans les mondes du spectacle vivant. De plus, les spectateurs tendent spontanément à concentrer leurs choix sur les propositions ayant la plus forte notoriété, ce qui pose la question délicate de l'adaptation des compagnies aux techniques du «marketing de l'offre » afin, dans un contexte de surabondance de l'offre, d'élargir l'audience des spectacles en captant l'attention des programmateurs et des spectateurs.

Un scénario en faveur de la diversité artistique et culturelle exigerait une volonté politique forte, soutenue et relayée par les professionnels du spectacle vivant, en faveur d'une démocratie renforcée dans ce domaine artistique. Elle devrait en particulier tenir pour décisif un rééquilibrage plus ferme des ressources symboliques et économiques disponibles au profit des différents acteurs qui font toute la richesse et la variété territoriales de cette filière d'activité.

\section{Des relations plus symétriques entre les artistes et les non-professionnels ?}

Une minorité de compagnies accordent une grande importance aux dispositifs de création en coproduction avec des personnes, sollicitées pour témoigner de leurs expériences de vie personnelle et en relation avec leur environnement social local. Cette association d'individus particuliers à une interrogation artistique sur leur univers de vie est susceptible d'ouvrir un champ de possibles émancipateur. Elle correspond aussi à une demande de participation culturelle active de la part de non-professionnels, attirés par des démarches créatives.

La production de ces compagnies est néanmoins souvent jugée de moindre qualité artistique par les experts publics, voire considérée comme relevant d'un travail social plutôt qu'artistique. Parallèlement, de nombreux auteurs de théâtre et certains chorégraphes souhaitent confronter leur écriture dramatique et scénique à leur perception du réel, comme en témoigne la référence aux guerres dans l'exYougoslavie dans les créations des années 1990. Le traitement artistique des matériaux ainsi recueillis pose la question de la mise en fiction des événements et des personnages ou de la restitution des témoignages en leur état brut, ou encore du degré d'articulation entre ces deux axes directeurs. La question des risques d'instrumentalisation sociale et politique des artistes est toujours aussi récurrente, ainsi que les marques d'hostilité d'une partie des milieux artistiques envers une démarche jugée populiste.

La stratification sociale persistante des publics des spectacles professionnels pose pourtant la question du soutien à de nouveaux types de mise en relation des personnes avec les œuvres, autant que celle de l'instauration de relations plus symétriques entre professionnels et amateurs. Les compagnies sont amenées à ne pas ignorer - et ce, dès la phase de conception - la réalité in fine incontournable de l'appropriation des publics, et donc la question de l'éventualité de leur participation aux différentes phases du processus et des modalités concrètes de cette coopération. Pourtant, le champ de recherche sur les apports des expériences artistiques en termes d'éveil émotionnel ou de sociabilité et de construction identitaire reste encore fort peu exploré.

Dans ce contexte, l'intégration de la rémunération des temps de transmission et de partage artistique et culturel entre artistes et non-professionnels (en particulier dans les milieux scolaires, associatifs...) comme revenu ordinaire des intermittents du spectacle vivant est souhaitée par plusieurs sociologues ${ }^{15}$ et des artistes qui ont développé leurs activités pédagogiques, tandis que d'autres artistes, à l'inverse, rejettent cette perspective, susceptible selon eux de dénaturer le cœur de leur identité professionnelle. PierreMichel Menger souligne par ailleurs un défaut de responsabilisation des employeurs du spectacle vivant vis-à-vis de la prise en charge du déficit du régime d'assurance-chômage spécifique des intermittents par la solidarité interprofessionnelle, et il y situe la source de la désintégration du marché du travail artistique ${ }^{16}$. La faible rémunération des interventions pédagogiques soulève aussi la question du taux de TVA qui leur est appliqué. Ne serait-il pas déjà judicieux de l'abaisser de 19,6\% à $7 \%$, puisque ces actions artistiques sont notamment destinées à élargir le public ?

\section{Des relations plus coopératives entre les professionnels?}

Georges Buisson a proposé en 2003 une redistribution des financements publics avec un retrait de l'État pour le financement des bâtiments culturels, reporté sur les collectivités territoriales, de façon à concentrer les aides de l'État

15. Voir Emmanuel WALlon, «L'éducation artistique», dans Philippe PoIRrIER, Politiques et pratiques de la culture, Paris, La Documentation Française, 2010 ; Philippe Henry, qui estime sur ce point et dans une note préparatoire au chapitre de conclusion de la recherche que « le développement des compagnies ne peut aller sans une reconsidération des équilibres professionnels et économiques, dont on sait qu'ils sont des compromis temporaires entre enjeux distincts et pour partie conflictuels ».

16. Pierre-Michel Menger, «Les professions culturelles : un système incomplet de relations sociales », dans Philippe PoIRrIER, op. cit., 2010. 
sur des « coopératives artistiques ». Elles seraient constituées d'une dizaine d'artistes environ, salariés pour une période de trois à quatre ans, autour d'une personnalité artistique $^{17}$.

Cette proposition s'inscrit dans la recherche d'une sécurisation professionnelle et entrepreneuriale par la restauration des conditions d'une permanence artistique, susceptible de relâcher la double pression concurrentielle du marché et de l'aide publique. Cette dernière, en effet, prioritairement tournée vers un soutien aux créations et la nécessité de nouer des relations partenariales avec des établissements artistiques ou culturels, exerce une pression conduisant à multiplier les créations. Pour une part induite par le milieu professionnel lui-même, cette logique de guichet financier contribue aussi à limiter les temps de recherche artistique des compagnies.

Il n'est pas sûr néanmoins que les perspectives de stabilisation des emplois artistiques intéressent une majorité d'intermittents. Désireux de rester créatifs et innovants, beaucoup d'entre eux sont attachés à une diversification des expériences professionnelles, en particulier grâce à une circulation interorganisationnelle. Ils souhaitent donc rester disponibles pour des opportunités de travail qui seraient source d'une rémunération symbolique et/ou financière diversifiée, voire plus élevée.

La double fonction risquée d'inventeur et d'entrepreneur, qui est celle des directeurs artistiques, pose la question de la reconnaissance sans ambiguïté du cumul d'une position d'entrepreneur de spectacles vivants et d'une possibilité d'accès aux droits sociaux de ces salariés au titre d'un emploi rémunéré intermittent, si le budget de la compagnie ne permet pas de financer un emploi à rémunération continue.

Une autre façon structurelle d'envisager l'instauration de rapports plus égalitaires au sein de la filière du spectacle vivant consiste à redistribuer la valeur ajoutée de son aval vers l'amont, de façon à offrir plus de moyens pour le financement de la fonction de recherche-expérimentation et de celle de création-production.

Plusieurs mécanismes existent déjà en ce sens. Les sociétés de perception et de répartition des droits d'auteurs et de droits voisins mutualisent le quart de la taxe sur la copie privée et les droits non répartis après dix années pour financer des aides, soit sociales, soit à la création essentiellement. L'Association de soutien des théâtres privés (ASTP) s'est créée en 1964 afin de constituer un fonds de mutualisation des risques, financé par une taxe de 3,5\% sur les recettes de billetterie et surtout mis en œuvre pour apporter une garantie en cas de déficit lors des trois premiers mois d'exploitation des spectacles. La création du Centre national de la chanson, des variétés et du jazz (CNV), par une scission au sein de l'ASTP en 1985, a maintenu le principe de ce fonds, qui est mieux alimenté dans un secteur plus soumis aux logiques de marché. Le déficit structurel des spectacles a rendu néanmoins nécessaire une intervention publique, qui a financé 45,3\% des fonds de l'ASTP en 2007, contre $2,7 \%$ pour le $\mathrm{CNV}^{18}$.

La généralisation d'une taxe sur la billetterie, la redistribution d'une partie accrue des droits d'auteurs, une réaffectation des impôts prélevés sur les activités indirectement liées au spectacle vivant pourraient constituer des fonds pour une gouvernance plus coopérative de la filière. Celle-ci pourrait également s'appuyer sur le développement d'agences régionales pour l'accompagnement des compagnies dans les différentes étapes de leur développement. Néanmoins, la perspective d'une extension de la taxe fiscale sur la billetterie des spectacles au secteur subventionné, suggérée lors des entretiens de Valois en 2009, a été repoussée notamment par les organismes de gestion mutualisée du secteur privé.

Sur un plan plus local, d'autres expérimentations voient le jour, comme le Créa'Fonds en Aquitaine ${ }^{19}$. Sur la base d'une expertise partagée et d'un fonds collectif, ce dispositif associe la mutualisation au moins partielle du risque inhérent à l'expérimentation et à la production artistiques et la mutualisation symétrique des succès et des bénéfices éventuels.

L'examen du monde du spectacle plaide donc en faveur d'une reconfiguration structurelle, de manière à ce qu'il soit plus et mieux articulé. Elle paraît chaque jour plus nécessaire pour faire face aux déséquilibres actuels et pour chercher à compenser les déstabilisations suscitées tout à la fois par la montée en puissance des logiques de marché et la difficulté des aides publiques à dégager de nouvelles marges au profit des organisations artistiques au-delà des plus prestigieuses et médiatisées. Les solutions envisageables suscitent néanmoins des débats encore vifs car elles interrogent les objectifs artistiques et culturels des compagnies, comme l'intérêt ou la faisabilité de démarches plus coopératives.

17. Georges Buisson, « Pour un renouveau du théâtre public », dans Cécil GuiTARD, la Bataille de l'imaginaire, Toulouse, Éd. de l'Attribut, 2009. 18. Daniel UrRutiaguer, Économie et droit du spectacle vivant en France, Paris, PSN, 2009, p. 23.

19. Le Créa'Fonds est « un collectif d'accompagnement technique et financier de la production dans le domaine du spectacle vivant». Fondé à l'initiative de collectivités locales, d'établissements culturels et d'une compagnie d'Aquitaine (Opéra Pagaï), il est également soutenu par plusieurs organismes financiers (dont la Fondation de France). Chacun participe à un fonds d'apport pour des productions de spectacle vivant. Les compagnies aidées reversent au fonds $2,5 \%$ du prix de vente des représentations des spectacles. 


\section{ÉLÉMENTS COMPLÉMENTAIRES CONCERNANT LES DEUX ÉCHANTILLONS DE COMPAGNIES}

\section{Échantillon qualitatif}

Les études de cas ont conduit à l'hypothèse centrale d'une différenciation des compagnies selon deux variables principales:

- d'abord la part relative de l'activité de diffusion de spectacles, dans la région d'appartenance de la compagnie et hors de celle-ci, qui peut en particulier se mesurer d'après la distribution territoriale des représentations;

- ensuite le type d'établissement (artistique ou non, labellisé ou non par le ministère de la Culture et de la Communication) dans lesquels cette diffusion s'opère, et le type d'établissement dans lesquels les compagnies effectuent l'essentiel de leurs activités d'action artistique et culturelle ou de formation.

Pour explorer cette intuition, un double indicateur synthétique et chiffré a été construit, qui a confirmé l'hypothèse.

Le premier indicateur mesure le pourcentage moyen sur trois ans $(2007,2008$ et 2009) de représentations de spectacles dans la région d'appartenance de la compagnie. II aboutit au repérage d'une distribution des compagnies sur l'axe linéaire correspondant («Taux de diffusion régionale en \% ", de 100 à $0 \%$ de représentations sur le territoire de la région d'appartenance). La part de la diffusion de spectacles à l'étranger est inversement corrélée à la diffusion dans la région d'appartenance.

Le second indicateur est obtenu par l'addition des pourcentages de diffusion des spectacles selon la stratification de quatre grands niveaux d'établissements :

$\mathrm{N} 1$ : établissements de production labellisés (EPCI nationaux, CDN, CDR ; CCN, CDC) et festivals internationaux (dont le In d'Avignon);

$\mathrm{N} 2$ : établissements de diffusion labellisés (scènes nationales, scènes conventionnées) et festivals nationaux;

N3 : établissements culturels de ville, salles parisiennes non labellisées, Off d'Avignon, festivals régionaux;

N4 : autres établissements (non artistiques).

Pour chaque compagnie, un « indicateur de stratification » a été constitué à partir de la somme des pourcentages moyens de ces quatre types d'établissement dans la diffusion de ses spectacles sur trois ans, pondérée par le rang institutionnel des lieux d'accueil. Le niveau $\mathrm{N} 4$, qui occupe le rang le moins prestigieux et a priori le moins rémunérateur, a ainsi été affecté d'un coefficient 1, le niveau N3 d'un coefficient 2, le niveau N2 d'un coefficient 3 et le niveau N1, au som- met de la hiérarchie institutionnelle, d'un coefficient 4. Cela donne une échelle allant de 100 (toute la distribution dans des équipements de type N4) à 400 (toute la distribution dans les équipements de type N1).

Ce principe a permis d'amplifier visuellement la distribution des différents cas étudiés sur un second axe ("Niveaux de stratification des établissements de diffusion »). Celui-ci n'exprime aucune échelle particulière de valeur quant à l'activité propre des compagnies. Elle rend simplement plus lisible leur mode d'inscription dans la hiérarchie encore actuellement en vigueur des établissements artistiques et culturels. La distribution générale sur le plan ainsi formé fait apparaître une courbe de tendance en diagonale, qui indique une corrélation certaine entre les deux indicateurs.

Le groupe A (compagnies dites «régionales » dont l'activité se déroule exclusivement ou presque dans leur région d'appartenance) est composé de sept cas principaux (A1 à $A 7)$, que complètent deux autres cas à budget plus important (A8) ou à diffusion exclusive en N2 (A9). Le dernier cas (A10) correspond à une compagnie disposant d'un important lieu de travail artistique mis à disposition par une municipalité.

Le groupe B (compagnies dites «transrégionales » dont l'activité reste encore majoritaire dans la région d'appartenance, mais où la diffusion hors de la région d'appartenance est déjà réelle) est composé de onze cas principaux ( $\mathrm{B} 1$ à $\mathrm{B} 11$ ), auxquels peuvent être reliés deux autres cas où la diffusion en $\mathrm{N} 2$ ou N1 s'avère plus importante (B13 et B14) et encore deux autres compagnies disposant d'un lieu de travail artistique mis à disposition par une municipalité (B12) ou mobile et acquis en propre (B15).

Le groupe C (compagnies dites «multirégionales » dont l'activité hors de la région d'appartenance - en France ou/et à l'étranger - est cette fois-ci majoritaire, même si une diffusion dans cette région reste sensible) est composé de neuf cas principaux ( $\mathrm{C} 1$ à $\mathrm{C} 9$ ), auxquels peuvent se rattacher trois cas plus atypiques. L'un se situe à l'articulation des «transrégionales » et de $\mathrm{C}(\mathrm{C} 10)$, un autre développe une activité moins importante que la moyenne du groupe (C11), le dernier disposant d'un lieu et d'une notoriété étendue (C12).

Le groupe D (compagnies dites «excentrées » dont l'activité se déroule essentiellement en dehors de leur région d'appartenance et où les établissements de diffusion sont majoritairement en N2 et N1) est composé de sept cas principaux (D1 à D7), auxquels peuvent être

\section{Graphique A - Typologie des compagnies}

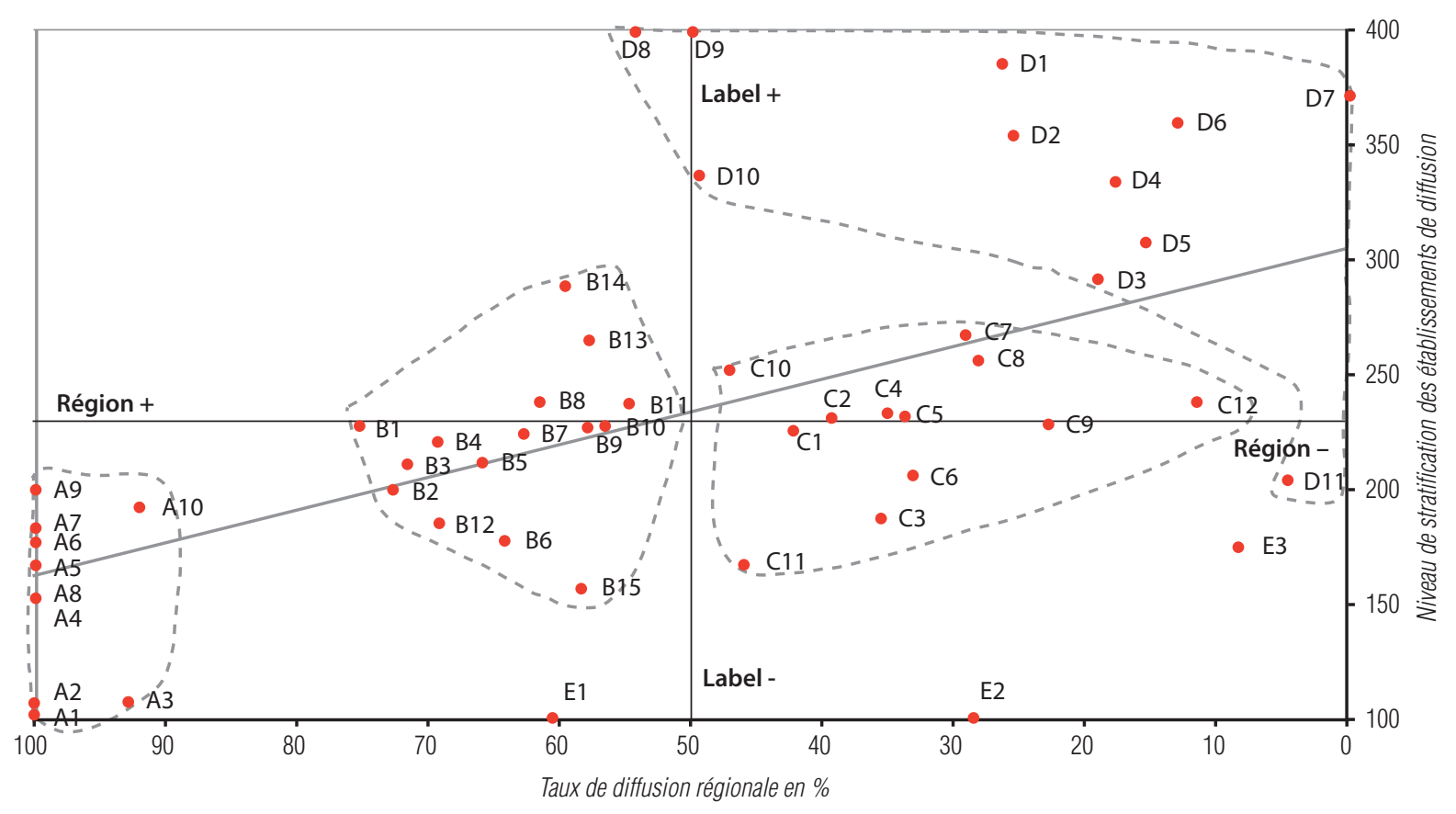


rattachés trois autres cas plus reliés à leur région d'appartenance (D8 à D10). Un dernier cas (D11) se caractérise par une très grande importance de la diffusion à l'étranger.

Enfin, trois derniers cas ne sont pas rattachables à la typologie précédente et constituent des cas d'espèce (E1 à E3).

Les deux lignes à trait plein tracées dans le graphique correspondent respectivement au taux de $50 \%$ de la diffusion des spectacles dans la région d'appartenance, pour l'axe horizontal des abscisses, et à la moyenne de l'indice de stratification des établissements, pour l'axe vertical des ordonnées.

\section{Échantillon quantitatif}

L'analyse en composantes principales est une méthode factorielle qui permet de réduire le nombre de caractères d'une population étudiée en sélectionnant ceux qui différencient le plus des groupes d'individus. Ces derniers sont ainsi rendus relativement plus homogènes par le partage de traits similaires, qui les différencient des autres groupes.

II s'agit de projeter les composantes principales, c'est-à-dire les caractères les plus discriminants des compagnies, sur un plan formé par un axe horizontal et un axe vertical. Dans le nuage de points représenté sur le graphique, les distances entre les caractères selon leur disposition autour des deux axes permettent de les classer en groupes. Le principe est de retenir les distances maximales entre les points du nuage pour repérer les traits des compagnies qui s'opposent le plus.

L'axe horizontal du graphique oppose principalement de gauche à droite :

- le rang du temps consacré par les compagnies à la diffusion de spectacles (Temps consacré à la diffusion) au rang du temps qu'elles dédient aux manifestations d'action culturelle et de formation professionnelle (Temps consacré à l'action culturelle);

- le rang de l'espace international dans le temps consacré à la diffusion

(Temps consacré à la diffusion à l'étranger) à celui de la région d'im- plantation dans le temps réservé à l'action culturelle et la formation professionnelle (Temps consacré à l'action culturelle dans sa région) - le rang de l'espace international dans les revenus des ventes de spectacles (Revenus issus de l'étranger) à celui de la région d'implantation dans ces revenus (Revenus de la région), ainsi qu'au rang des revenus de l'action culturelle et de la formation professionnelle dans le total des revenus (Revenus de l'action culturelle).

Cet axe oppose, dans une moindre mesure, parmi les compagnies qui bénéficient d'un financement public croisé (cumul de subventions publiques accordées par l'État et par des collectivités territoriales), celles pour lesquelles les subventions étatiques sont supérieures aux dotations des collectivités territoriales (Subventions État majoritaires, à gauche) à celles qui sont dans la situation inverse (Subventions collectivités territoriales majoritaires, à droite). L'intensité de la différenciation est du même ordre entre les compagnies chorégraphiques (Danse, à gauche mais assez proche du centre du graphique) et les troupes théâtrales (Théâtre, à droite et plus en haut).

L'axe vertical du graphique oppose surtout de haut en bas:

- le niveau du budget, mesuré par le total des charges (Budget), à l'année de création des compagnies (Année de création);

- le rang des subventions globales dans le total des revenus (Subvention) à l'absence de subventions des collectivités publiques nationales (Aucune);

- les compagnies dirigées par un homme (Direction artistique $\widehat{0}$ ) à celles qui le sont par une femme (Direction artistique $q$ ).

Le point représentant le nombre de contrats à durée indéterminée déclaré par les compagnies (CDI) a une position proche de celui du total des charges (Budget) dans la partie supérieure gauche du graphique. Cela confirme que la stabilisation des emplois des compagnies est dépendante de leur niveau de budget. Le point correspondant aux compagnies qui n'ont pas déclaré d'action culturelle ou de formation professionnelle (Aucune action culturelle) est situé vers le bas de la carte.

\section{Graphique B - Les deux premiers axes de l'analyse en composantes principales de l'échantillon quantitatif (enquête par questionnaire)}

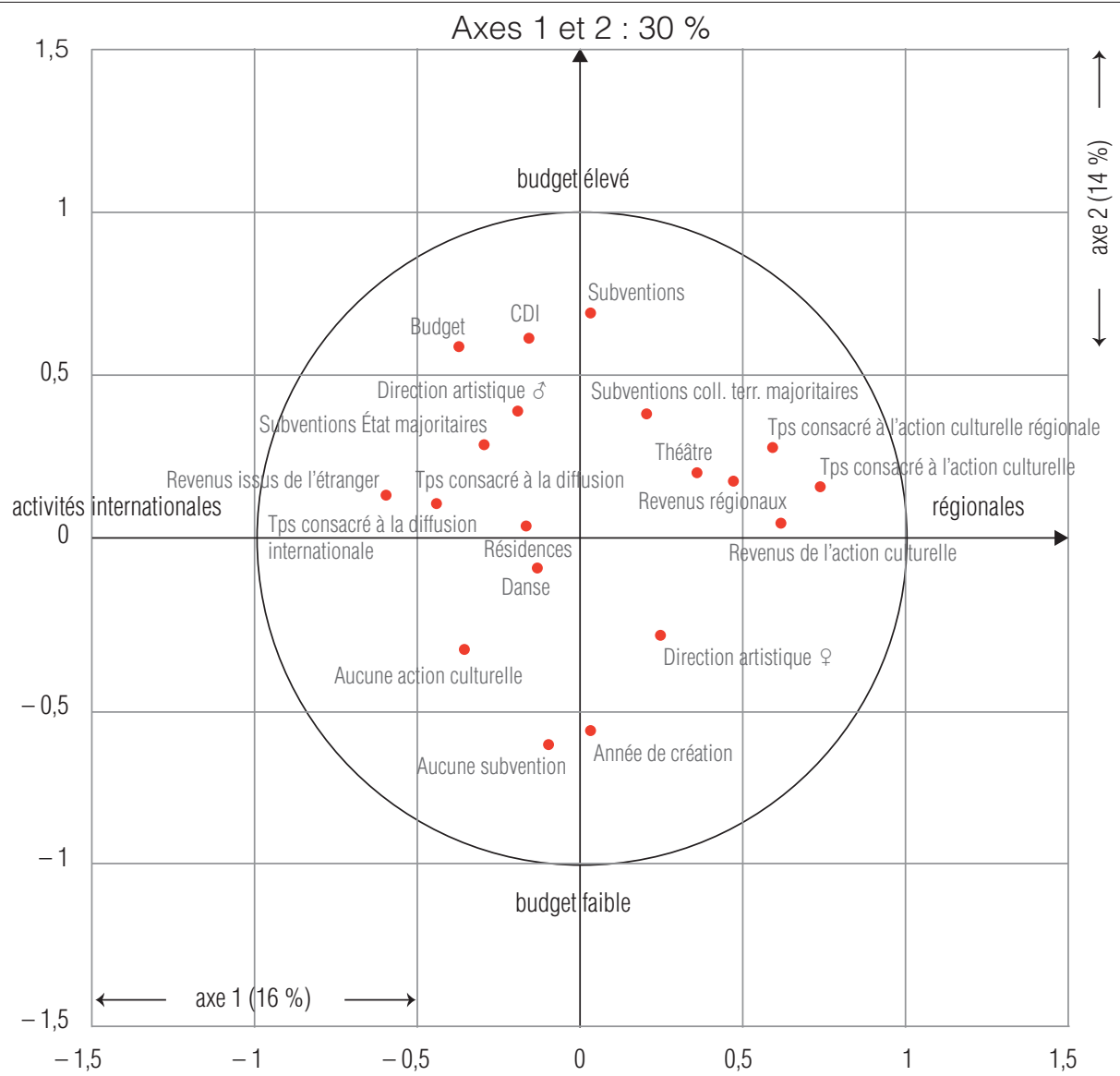




\section{Éléments de méthodologie}

La démarche a consisté à croiser les méthodes quantitative et qualitative d'enquête.

51 études de cas approfondies ont été réalisées, à partir d'un guide d'enquête sériant divers documents budgétaires et d'activité précis et généralement disponibles, sur les saisons 2007 à 2009, ainsi que différents points à aborder dans un entretien semi-directif avec le directeur artistique et/ou l'administrateur de chaque compagnie.

Un questionnaire sur les ressources et les activités en 2009 a été envoyé à l'ensemble des compagnies qui disposaient d'une adresse électronique valide en octobre 2010, en particulier grâce à l'appui de différents offices régionaux pour la culture. II a permis de constituer un échantillon représentatif de 572 réponses. Le taux de couverture selon les régions varie de 9 à $30 \%$, avec une moyenne nationale de presque $15 \%$. La méthodologie du questionnaire, fondée surtout sur des questions ordinales à propos du rang des temps consacrés aux différentes activités, des types de ressources, enfin des territoires de rayonnement (régional, national, international) pour les deux dimensions précédentes, s'est montrée aussi opérationnelle que les données plus précisément quantifiées dans les études de cas approfondies.

Le champ territorial a été circonscrit à la France métropolitaine. L'échantillon quantitatif est concentré sur les disciplines liées au théâtre $(48 \%)$ et à la danse $(19 \%)$, en incluant les arts du cirque $(6 \%)$, de la rue $(8 \%)$, de la marionnette $(6 \%)$ et du conte $(3 \%)$, tandis que $10 \%$ des compagnies se définissent comme pluridisciplinaires. Les ensembles musicaux et vocaux n'ont pas été traités, ni les artistes indépendants, ni les compagnies qui ont géré un établissement labellisé entre 2007 et 2009.

Une première analyse qualitative des études de cas a permis de dégager plusieurs hypothèses de travail et d'orienter les traitements statistiques à opérer prioritairement. La méthode de l'analyse par composantes principales (ACP) sur les deux échantillons a alors permis de confirmer et préciser ces hypothèses. Les nombreuses appréciations des personnes interrogées ont également conduit à dégager des éléments de contextualisation sur les démarches passées et envisagées. Mais les représentations des dirigeants sur leurs pratiques et l'image de l'organisation qu'ils construisent dans leur discours sont confrontées à des données quantitatives. Celles-ci permettent de dégager des éléments minorés ou appréhendés de façon un peu floue par les interlocuteurs.

L'interprétation générale a été effectuée en partant d'abord des éléments factuels et des récurrences qui se dégagaient des données rassemblées sur nos deux échantillons. Elle s'est poursuivie par une problématisation de ces données dans le cadre d'une approche d'analyse de filière. La mise en relation avec certains éléments contextuels propres au spectacle vivant et aux politiques publiques associées a enfin dégagé plusieurs questions portant sur l'avenir des compagnies dans l'environnement qui est aujourd'hui le leur.

\section{RÉSUMÉ}

Quel lien peut-on établir entre la distribution territoriale des différentes activités des compagnies théâtrales et chorégraphiques en France et la structure de leurs ressources, monétaires et non financières ? À partir de deux échantillons de compagnies et en s'appuyant sur la littérature consacrée au sujet, la recherche permet de souligner l'importance, pour le développement des compagnies, non seulement de leur région d'appartenance ou d'implantation, mais aussi des établissements artistiques et culturels non labellisés par le ministère de la Culture et de la Communication. Elle précise la place centrale, mais aussi la situation problématique des compagnies dans la filière actuelle du spectacle vivant. Elle apporte des arguments pour une nécessaire reconfiguration structurelle du partenariat entre privé et public sur lequel cette filière s'est particulièrement développée depuis la fin du $\mathrm{Xx}^{\mathrm{e}}$ siècle.

\section{ABSTRACT}

This research on French theatrical and choreographic companies deals with the link between the distribution of their different activities and the structure of their ressources, whether monetary or non monetary. It is based both on two samples and the material available on the subject. We highlight how the region in which they are established along with the establishments without any national label are important for their development. We make clear the central role but also the paradoxical situation of the companies in the current performing arts value chain. We provide arguments in favour of a necessary new structural reconfiguration of the private/public relationship through which this value chain has particularly developed itself since the end of the $20^{\text {th }}$ century.

\section{Tous les documents publiés par le DEPS sont téléchargeables sur http://www.culturecommunication.gouv.fr/deps et sur www.cairn.info}

Le DEPS n'assurant pas de diffusion physique de ses collections, nous vous proposons de vous informer régulièrement des parutions par message électronique. Pour ce faire, merci de bien vouloir nous communiquer votre courriel à l'adresse contact.deps@culture.gouv.fr 